\title{
INSTANTON COUNTING ON BLOWUP. II. $K$-THEORETIC PARTITION FUNCTION
}

\author{
HIRAKU NAKAJIMA AND KŌTA YOSHIOKA \\ Dedicated to Vladimir Drinfeld on his fiftieth birthday
}

\begin{abstract}
We study Nekrasov's deformed partition function $Z\left(\varepsilon_{1}, \varepsilon_{2}, \vec{a} ; \mathfrak{q}, \boldsymbol{\beta}\right)$ of 5-dimensional supersymmetric Yang-Mills theory compactified on a circle. Mathematically it is the generating function of the characters of the coordinate rings of the moduli spaces of instantons on $\mathbb{R}^{4}$. We show that it satisfies a system of functional equations, called blowup equations, whose solution is unique. As applications, we prove (a) $F\left(\varepsilon_{1}, \varepsilon_{2}, \vec{a} ; \mathfrak{q}, \boldsymbol{\beta}\right)=\varepsilon_{1} \varepsilon_{2} \log Z\left(\varepsilon_{1}, \varepsilon_{2}, \vec{a} ; \mathfrak{q}, \boldsymbol{\beta}\right)$ is regular at $\varepsilon_{1}=\varepsilon_{2}=0$ (a part of Nekrasov's conjecture), and (b) the genus 1 parts, which are first several Taylor coefficients of $F\left(\varepsilon_{1}, \varepsilon_{2}, \vec{a} ; \mathfrak{q}, \boldsymbol{\beta}\right)$, are written explicitly in terms of $\tau=d^{2} F(0,0, \vec{a} ; \mathfrak{q}, \boldsymbol{\beta}) / d a^{2}$ in rank 2 case.
\end{abstract}

\section{INTRODUCTION}

In Part I of this paper [13, we studied Nekrasov's partition function [15] for $\mathcal{N}=2$ supersymmetric gauge theory in 4-dimension (see also [14]). It is defined as the generating function of the integral of the equivariant cohomology class 1 of the framed moduli space $M(r, n)$ of torsion free sheaves on $\mathbb{P}^{2}$ with rank $r, c_{2}=n$ :

$$
Z^{\text {inst }}\left(\varepsilon_{1}, \varepsilon_{2}, \vec{a} ; \mathfrak{q}\right)=\sum_{n=0}^{\infty} \mathfrak{q}^{n} \int_{M(r, n)} 1 .
$$

Here $(r+2)$-dimensional torus $\widetilde{T}$ acts naturally on $M(r, n)$, and $\varepsilon_{1}, \varepsilon_{2}, \vec{a}=\left(a_{1}, \ldots, a_{r}\right)$ are generators of $H_{\widetilde{T}}^{*}(\mathrm{pt})=S^{*}(\operatorname{Lie} \widetilde{T})$. (More precisely, this is the instanton part of the partition function. We multiply it with the perturbative part. See \$4.2]) It can be considered as series of equivariant Donaldson invariants for $\mathbb{R}^{4}$, and there are close relation to the ordinary Donaldson invariants, such as blowup formulas, wall-crossing formulas [14, 5].

In this part II, we study a similar partition function, in which we replace the integration in the equivariant cohomology by one in equivariant $K$-theory:

$$
Z^{\text {inst }}\left(\varepsilon_{1}, \varepsilon_{2}, \vec{a} ; \mathfrak{q}, \boldsymbol{\beta}\right)=\sum_{n}\left(\mathfrak{q} \boldsymbol{\beta}^{2 r} e^{-r \boldsymbol{\beta}\left(\varepsilon_{1}+\varepsilon_{2}\right) / 2}\right)^{n} \sum_{i}(-1)^{i} \operatorname{ch} H^{i}(M(r, n), \mathcal{O}) .
$$

We consider $e^{\boldsymbol{\beta} a_{\alpha}}, e^{\boldsymbol{\beta} \varepsilon_{1}}, e^{\boldsymbol{\beta} \varepsilon_{2}}$ as characters of $\widetilde{T}$ here. The formal parameter $\boldsymbol{\beta}$ is introduced so that the $K$-theoretic partition function converges to the homological one when $\boldsymbol{\beta} \rightarrow 0$. It is called the partition function of the 5-dimensional supersymmetric gauge theory compactified on a circle in the physics literature, where the radius of the circle is $\boldsymbol{\beta}$.

2000 Mathematics Subject Classification. Primary 14D21; Secondary 57R57, 81T13, 81T60.

The first author is supported by the Grant-in-aid for Scientific Research (No.13640019, 15540023), JSPS. 
Nekrasov [15] conjectured that $F^{\text {inst }}\left(\varepsilon_{1}, \varepsilon_{2}, \vec{a} ; \mathfrak{q}\right)=\varepsilon_{1} \varepsilon_{2} \log Z^{\text {inst }}\left(\varepsilon_{1}, \varepsilon_{2}, \vec{a} ; \mathfrak{q}\right)$ is regular at $\varepsilon_{1}, \varepsilon_{2}=0$, and $F_{0}^{\text {inst }}(\vec{a} ; \mathfrak{q}) \stackrel{\text { def. }}{=} F^{\text {inst }}(0,0, \vec{a} ; \mathfrak{q})$ is the instanton part of the Seiberg-Witten prepotential for $\mathcal{N}=2$ supersymmetric gauge theory [17] with gauge group $\mathrm{SU}(r)$. The SeibergWitten prepotential is defined by certain period integrals of hyperelliptic curves, the so-called Seiberg-Witten curves. (See 14 for detail.) This was a mathematically well formulated conjecture, which is similar to the mirror symmetry. The conjecture for the homological partition function was proved affirmatively by the authors [13] and Nekrasov-Okounkov [16] independently. Nekrasov also conjectured the same statements for the above $K$-theoretic version, and the technique in [16] can be applied to that case also.

We study the $K$-theoretic partition function through the approach taken in [13, which we briefly describe now: We consider similar correlation functions given by generating functions of characters of cohomology groups of Donaldson divisors $\mu(C)$ on the moduli spaces $\widehat{M}(r, k, n)$ on the blowup plane $\widehat{\mathbb{P}}^{2}$. As a simple application of the Atiyah-Bott-Lefschetz formula, the correlation functions can be expressed by $Z^{\text {inst }}$. (See (2.2).) On the other hand, through a geometric study of moduli spaces on the blowup, we prove vanishing of certain cohomology groups. (See Theorem 2.4.) Combining these two results, we get a system of functional equations, called blowup equations, satisfied by $Z^{\text {inst }}$. It determines the coefficients of $\mathfrak{q}^{n}$ in $Z^{\text {inst }}$ recursively. It also implies the regularity of $F^{\text {inst }}\left(\varepsilon_{1}, \varepsilon_{2}, \vec{a} ; \mathfrak{q}, \boldsymbol{\beta}\right)$ and we get a differential equation for $F_{0}^{\text {inst }}(\vec{a} ; \mathfrak{q}, \boldsymbol{\beta})$, as limits of the blowup equations. (See (4.17).) We call it a contact term equation, according to the name of their homological version. The contact term equation also determines $F_{0}^{\text {inst }}(\vec{a} ; \mathfrak{q}, \boldsymbol{\beta})$ recursively.

The homological version of the contact term equation was much studied in the physics literature, and the Seiberg-Witten prepotential satisfies the equations (see [6, 10] and the reference therein). By the uniqueness of its solution, the instanton part of the Seiberg-Witten prepotential is equal to $F_{0}^{\text {inst }}(\vec{a} ; \mathfrak{q})$. This was our proof of Nekrasov's conjecture in [13]. It is natural to hope that the same proof can be given for the $K$-theoretic version. But we do not find our $K$-theoretic contact term equation in the physics literature, and do not know how to prove this assertion at this moment except for $r=2$ case.

Although we do not give the proof of Nekrasov's conjecture, we think that it is worthwhile to pursue our approach by various reasons:

(1) The blowup equations determine not only $F_{0}^{\text {inst }}(\vec{a} ; \mathfrak{q}, \boldsymbol{\beta})$, but also several higher coefficients of the expansion of $F^{\text {inst }}\left(\varepsilon_{1}, \varepsilon_{2}, \vec{a} ; \mathfrak{q}, \boldsymbol{\beta}\right)$ at $\varepsilon_{1}=\varepsilon_{2}=0$. (See \$5.)

(2) The geometric study of moduli spaces on the blowup is probably useful for the study of the $K$-theoretic version of Donaldson invariants.

Higher coefficients are identified with higher genus Gromov-Witten invariants for certain noncompact Calabi-Yau 3-folds (see [15, 14, §7]), and appear in the wall-crossing formula for Donaldson invariants [5]. Thus they are equally important as $F_{0}^{\text {inst }}(\vec{a} ; \mathfrak{q}, \boldsymbol{\beta})$.

For the ordinary Donaldson invariants, the vanishing of first several blowup coefficients was well-known, and was proved by the dimension counting argument. Our proof of the blowup equation for the homological partition function was given by the same idea. The proof for the $K$-theoretic partition function is very different, and we use the Kawamata-Viewheg vanishing theorem, a result from complex algebraic geometry. But we hope that a similar result holds for the $K$-theoretic version of Donaldson invariants, whose existence is still conjectural.

Acknowledgement. The authors are grateful to the referee for helpful suggestions and comments. 


\section{1. $K$-ThEORETIC PARTITION FUnCTION}

In this section, we define the $K$-theoretic version of Nekrasov's partition function. We follow [13, 14 for which the reader can find more detail and the references.

1.1. Definition of the partition function. Let $M(r, n)$ denote the framed moduli spaces of torsion free sheaves $(E, \Phi)$ on $\mathbb{P}^{2}$ with rank $r$ and $c_{2}=n$. Let $M_{0}^{\mathrm{reg}}(r, n)$ be the open subvariety consisting of locally free sheaves. Let $M_{0}(r, n)$ be the Uhlenbeck (partial) compactification of $M^{\mathrm{reg}}(r, n)$, i.e.,

$$
M_{0}(r, n)=\bigsqcup_{n^{\prime}=0}^{n} M_{0}^{\mathrm{reg}}\left(r, n^{\prime}\right) \times S^{n-n^{\prime}} \mathbb{C}^{2} .
$$

We can endow this space with the structure of an affine algebraic variety so that there is a projective morphism

$$
\pi: M(r, n) \rightarrow M_{0}(r, n) .
$$

The corresponding map between closed points can be identified with

$$
(E, \Phi) \longmapsto\left(\left(E^{\vee \vee}, \Phi\right), \operatorname{Supp}\left(E^{\vee \vee} / E\right)\right) \in M_{0}^{\mathrm{reg}}\left(r, n^{\prime}\right) \times S^{n-n^{\prime}} \mathbb{C}^{2}
$$

where $E^{\vee \vee}$ is the double dual of $E$ and $\operatorname{Supp}\left(E^{\vee \vee} / E\right)$ is the support of $E^{\vee \vee} / E$ counted with multiplicities.

Let $T$ be the maximal torus of $\mathrm{GL}_{r}(\mathbb{C})$ consisting of diagonal matrices and let $\widetilde{T}=\mathbb{C}^{*} \times$ $\mathbb{C}^{*} \times T$. We define an action of $\widetilde{T}$ on $M(r, n)$ as follows: For $\left(t_{1}, t_{2}\right) \in \mathbb{C}^{*} \times \mathbb{C}^{*}$, let $F_{t_{1}, t_{2}}$ be an automorphism of $\mathbb{P}^{2}$ defined by

$$
F_{t_{1}, t_{2}}\left(\left[z_{0}: z_{1}: z_{2}\right]\right)=\left[z_{0}: t_{1} z_{1}: t_{2} z_{2}\right] .
$$

For $\operatorname{diag}\left(e_{1}, \ldots, e_{r}\right) \in T$ let $G_{e_{1}, \ldots, e_{r}}$ denote the isomorphism of $\mathcal{O}_{\ell_{\infty}}^{\oplus r}$ given by

$$
\mathcal{O}_{\ell_{\infty}}^{\oplus r} \ni\left(s_{1}, \ldots, s_{r}\right) \longmapsto\left(e_{1} s_{1}, \ldots, e_{r} s_{r}\right) .
$$

Then for $(E, \Phi) \in M(r, n)$, we define

$$
\left(t_{1}, t_{2}, e_{1}, \ldots, e_{r}\right) \cdot(E, \Phi)=\left(\left(F_{t_{1}, t_{2}}^{-1}\right)^{*} E, \Phi^{\prime}\right),
$$

where $\Phi^{\prime}$ is the composite of homomorphisms

$$
\left.\left(F_{t_{1}, t_{2}}^{-1}\right)^{*} E\right|_{\ell_{\infty}} \stackrel{\left(F_{t_{1}, t_{2}}^{-1}\right)^{*} \Phi}{\longrightarrow}\left(F_{t_{1}, t_{2}}^{-1}\right)^{*} \mathcal{O}_{\ell_{\infty}}^{\oplus r} \longrightarrow \mathcal{O}_{\ell_{\infty}}^{\oplus r} \stackrel{G_{e_{1}, \ldots, e_{r}}}{\longrightarrow} \mathcal{O}_{\ell_{\infty}}^{\oplus r} .
$$

Here the middle arrow is the homomorphism given by the action.

In a similar way, we have a $\widetilde{T}$-action on $M_{0}(r, n)$. The map $\pi: M(r, n) \rightarrow M_{0}(r, n)$ is equivariant.

Notation 1.2. We denote by $e_{\alpha}(\alpha=1, \ldots, r)$ the one dimensional $\widetilde{T}$-module given by

$$
\widetilde{T} \ni\left(t_{1}, t_{2}, e_{1}, \ldots, e_{r}\right) \mapsto e_{\alpha}
$$

Similarly, $t_{1}, t_{2}$ denote one-dimensional $\widetilde{T}$-modules. Thus the representation $\operatorname{ring} R(\widetilde{T})$ is isomorphic to $\mathbb{Z}\left[t_{1}^{ \pm}, t_{2}^{ \pm}, e_{1}^{ \pm}, \ldots, e_{r}^{ \pm}\right]$, where $e_{\alpha}^{-1}$ is the dual of $e_{\alpha}$.

We denote the coordinates of $\operatorname{Lie}(\widetilde{T})$ by $\varepsilon_{1}, \varepsilon_{2}, a_{1}, \ldots, a_{r}$ corresponding to $t_{1}, t_{2}, e_{1}, \ldots, e_{r}$. In our previous paper [13], these are generators of the equivariant cohomology group $H_{\widetilde{T}}^{*}(p t)$ of a single point. We relate two sets of variables as $t_{1}=e^{\boldsymbol{\beta} \varepsilon_{1}}, t_{2}=e^{\boldsymbol{\beta} \varepsilon_{2}}, e_{\alpha}=e^{\boldsymbol{\beta} a_{\alpha}}$, where $\boldsymbol{\beta}$ is a parameter. We will define the $K$-theory partition function so that it converges to the homological partition function when $\boldsymbol{\beta} \rightarrow 0$. 
We define the instanton part of the partition function by

$$
\begin{gathered}
Z^{\text {inst }}\left(\varepsilon_{1}, \varepsilon_{2}, \vec{a} ; \mathfrak{q}, \boldsymbol{\beta}\right) \stackrel{\text { def. }}{=} \sum_{n}\left(\mathfrak{q} \boldsymbol{\beta}^{2 r} e^{-r \boldsymbol{\beta}\left(\varepsilon_{1}+\varepsilon_{2}\right) / 2}\right)^{n} Z_{n}\left(\varepsilon_{1}, \varepsilon_{2}, \vec{a} ; \boldsymbol{\beta}\right) \\
Z_{n}\left(\varepsilon_{1}, \varepsilon_{2}, \vec{a} ; \boldsymbol{\beta}\right) \stackrel{\text { def. }}{=} \sum_{i}(-1)^{i} \operatorname{ch} H^{i}(M(r, n), \mathcal{O}) .
\end{gathered}
$$

Here the character ch is a formal sum of weight spaces, which are finite dimensional as shown in [13, §4]. We also have

$$
Z_{n}\left(\varepsilon_{1}, \varepsilon_{2}, \vec{a} ; \boldsymbol{\beta}\right)=\sum_{i}(-1)^{i} \operatorname{ch} H^{0}\left(M_{0}(r, n), R^{i} \pi_{*} \mathcal{O}\right) .
$$

We will see that the higher direct image sheaves $R^{i} \pi_{*} \mathcal{O}=0$ for $i>0$ (Lemma 3.1). Therefore we have

$$
Z_{n}\left(\varepsilon_{1}, \varepsilon_{2}, \vec{a} ; \boldsymbol{\beta}\right)=\operatorname{ch} H^{0}\left(M_{0}(r, n), \mathcal{O}\right) .
$$

This definition has an advantage that it involves only the Uhlenbeck compactification $M_{0}(r, n)$.

1.2. Other descriptions of the partition function. Let $K^{\widetilde{T}}(M(r, n))$ denote the Grothendieck group of $\widetilde{T}$-equivariant coherent sheaves on $M(r, n)$ and similarly for $K^{\widetilde{T}}\left(M_{0}(r, n)\right)$. These are modules over the representation ring $R(\widetilde{T})$ of the torus $\widetilde{T}$. As in 1.2 , we identify it with the Laurent polynomial ring $\mathbb{Z}\left[t_{1}^{ \pm}, t_{2}^{ \pm}, e_{1}^{ \pm}, \ldots, e_{r}^{ \pm}\right]$. Since $M(r, n)$ is nonsingular, $K^{\widetilde{T}}(M(r, n))$ is isomorphic to the Grothendieck group of $\widetilde{T}$-equivariant vector bundles. The proper equivariant morphism $\pi$ induces a homomorphism $\pi_{*}: K^{\widetilde{T}}(M(r, n)) \rightarrow K^{\widetilde{T}}\left(M_{0}(r, n)\right)$ by taking the alternating sum of higher direct image sheaves $\sum_{i}(-1)^{i} R^{i} \pi_{*}$.

The fixed points $M_{0}(r, n)^{\widetilde{T}}$ consist of the single point $n[0] \in S^{n} \mathbb{C}^{2} \subset M_{0}(r, n)$. Let $\iota_{0}$ denote the inclusion map of the fixed point. By the localization theorem for the K-theory due to Thomason [18] (a prototype was given in [1]), it is known that the homomorphism $\iota_{0 *}$ is an isomorphism after the localization:

$$
\iota_{0 *}: \mathcal{R} \cong K^{\widetilde{T}}\left(M_{0}(r, n)^{\widetilde{T}}\right) \otimes_{R(\widetilde{T})} \mathcal{R} \cong K^{\widetilde{T}}\left(M_{0}(r, n)\right) \otimes_{R(\widetilde{T})} \mathcal{R},
$$

where $\mathcal{R}=\mathbb{Q}\left(t_{1}, t_{2}, e_{1}, \ldots, e_{m}\right)$ is the quotient field of $R(\widetilde{T})$. We have $\iota_{0 *}^{-1}=$ ch, which is a consequence of a trivial identity $\mathrm{ch} \circ \iota_{0 *}=\mathrm{id}$. We thus get

$$
Z_{n}\left(\varepsilon_{1}, \varepsilon_{2}, \vec{a} ; \boldsymbol{\beta}\right)=\left(\iota_{0 *}\right)^{-1} \pi_{*}\left(\mathcal{O}_{M(r, n)}\right) .
$$

Here we denote the element in $K^{\widetilde{T}}(M(r, n))$ corresponding to $\mathcal{O}_{M(r, n)}$ by the same symbol for brevity. We hope that these two meanings can be distinguished from the content.

The fixed points $M(r, n)^{\widetilde{T}}$ consist of $(E, \Phi)=\left(I_{1}, \Phi_{1}\right) \oplus \cdots \oplus\left(I_{r}, \Phi_{r}\right)$ such that

a) $I_{\alpha}$ is an ideal sheaf of 0 -dimensional subscheme $Z_{\alpha}$ contained in $\mathbb{C}^{2}=\mathbb{P}^{2} \backslash \ell_{\infty}$.

b) $\Phi_{\alpha}$ is an isomorphism from $\left(I_{\alpha}\right)_{\ell_{\infty}}$ to the $\alpha$ th factor of $\mathcal{O}_{\ell_{\infty}}^{\oplus r}$.

c) $I_{\alpha}$ is fixed by the action of $\mathbb{C}^{*} \times \mathbb{C}^{*}$, coming from that on $\mathbb{P}^{2}$.

We parametrize the fixed point set $M(r, n)^{\widetilde{T}}$ by an $r$-tuple of Young diagrams $\vec{Y}=\left(Y_{1}, \ldots, Y_{r}\right)$ so that the ideal $I_{\alpha}$ is spanned by monomials $x^{i} y^{j}$ placed at $(i-1, j-1)$ outside $Y_{\alpha}$. The constraint is that the total number of boxes $|\vec{Y}| \stackrel{\text { def. }}{=} \sum_{\alpha}\left|Y_{\alpha}\right|$ is equal to $n$.

Let $\iota$ denote the inclusion map of the fixed point set. We have

$$
\iota_{*}: \bigoplus_{\vec{Y}} \mathcal{R} \cong K^{\widetilde{T}}\left(M(r, n)^{\widetilde{T}}\right) \otimes_{R(\widetilde{T})} \mathcal{R} \cong K^{\widetilde{T}}(M(r, n)) \otimes_{R(\widetilde{T})} \mathcal{R} .
$$


We then have

$$
Z_{n}\left(\varepsilon_{1}, \varepsilon_{2}, \vec{a} ; \boldsymbol{\beta}\right)=\sum_{\vec{Y}}\left(\iota_{*}\right)^{-1}\left(\mathcal{O}_{M(r, n)}\right)
$$

As $M(r, n)$ is nonsingular, $\left(\iota_{*}\right)^{-1}$ can be explicitly given by Atiyah-Bott Lefschetz fixed points formula:

$$
\iota_{*}^{-1}(\bullet)=\bigoplus_{\vec{Y}} \frac{\iota_{\vec{Y}}^{*}(\bullet)}{\bigwedge_{-1} T_{\vec{Y}}^{*} M(r, n)}
$$

where $T_{\vec{Y}}^{*} M(r, n)$ is the cotangent bundle of $M(r, n)$ at a fixed point of $\vec{Y}$ considered as a $\widetilde{T}$ module, $\bigwedge_{-1}$ is the alternating sum of exterior powers, and $\iota_{\vec{Y}}^{*}$ is the pull-back homomorphism with respect to the inclusion $\iota_{\vec{Y}}:\{\vec{Y}\} \rightarrow M(r, n)$. Here the pull-back homomorphism is defined via the isomorphism of $K^{\widetilde{T}}(M(r, n))$ and the Grothendieck group of $\widetilde{T}$-equivariant locally free sheaves.

In order to express $\bigwedge_{-1} T_{\vec{Y}}^{*}(M(r, n))$, we need some notation for Young diagrams. Let $Y=$ $\left(\lambda_{1} \geq \lambda_{2} \geq \cdots\right)$ be a Young diagram, where $\lambda_{i}$ is the length of the $i$ th column. Let $Y^{\prime}=\left(\lambda_{1}^{\prime} \geq\right.$ $\left.\lambda_{2}^{\prime} \geq \ldots\right)$ be the transpose of $Y$. Thus $\lambda_{j}^{\prime}$ is the length of the $j$ th row of $Y$. Let $l(Y)$ denote the number of columns of $Y$, i.e., $l(Y)=\lambda_{1}^{\prime}$. Let

$$
a_{Y}(i, j)=\lambda_{i}-j, \quad l_{Y}(i, j)=\lambda_{j}^{\prime}-i .
$$

Here we set $\lambda_{i}=0$ when $i>l(Y)$. Similarly $\lambda_{j}^{\prime}=0$ when $j>l\left(Y^{\prime}\right)$. When the square $s=(i, j)$ lies in $Y$, these are called arm-length, leg-length, respectively in the literature. But our formula below involves these also for squares outside $Y$. So these take negative values in general.

By [13, Theorem 2.11] we have

$$
Z^{\text {inst }}\left(\varepsilon_{1}, \varepsilon_{2}, \vec{a} ; \mathfrak{q}, \boldsymbol{\beta}\right)=\sum_{\vec{Y}} \frac{\left(\mathfrak{q} \boldsymbol{\beta}^{2 r} e^{-r \boldsymbol{\beta}\left(\varepsilon_{1}+\varepsilon_{2}\right) / 2}\right)^{|\vec{Y}|}}{\bigwedge_{-1} T_{\vec{Y}}^{*} M(r, n)}=\sum_{\vec{Y}} \frac{\left(\mathfrak{q} \boldsymbol{\beta}^{2 r} e^{-r \boldsymbol{\beta}\left(\varepsilon_{1}+\varepsilon_{2}\right) / 2}\right)^{|\vec{Y}|}}{\prod_{\alpha, \beta} n_{\alpha, \beta}^{\vec{Y}}\left(\varepsilon_{1}, \varepsilon_{2}, \vec{a} ; \boldsymbol{\beta}\right)}
$$

where

$$
\begin{aligned}
& n_{\alpha, \beta}^{\vec{Y}}\left(\varepsilon_{1}, \varepsilon_{2}, \vec{a} ; \boldsymbol{\beta}\right)=\prod_{s \in Y_{\alpha}}\left(1-e^{-\boldsymbol{\beta}\left(-l_{Y_{\beta}}(s) \varepsilon_{1}+\left(a_{Y_{\alpha}}(s)+1\right) \varepsilon_{2}+a_{\beta}-a_{\alpha}\right)}\right) \\
& \times \prod_{t \in Y_{\beta}}\left(1-e^{-\boldsymbol{\beta}\left(\left(l_{Y_{\alpha}}(t)+1\right) \varepsilon_{1}-a_{Y_{\beta}}(t) \varepsilon_{2}+a_{\beta}-a_{\alpha}\right)}\right) .
\end{aligned}
$$

Remark 1.5. Contrary to the convention in [13. Remark 4.4], we put the $\widetilde{T}$-module structure on the coordinate ring (and the cohomology groups) by $F_{g^{-1}}^{*}$, where $F_{g}: M(r, n) \rightarrow M(r, n)$ is the isomorphism given by an element $g \in \widetilde{T}$.

This combinatorial expression was the original definition of the instanton part of the partition function due to Nekrasov 15, except we put the additional factor $e^{-r \boldsymbol{\beta}\left(\varepsilon_{1}+\varepsilon_{2}\right) / 2}$. This factor is the half of the canonical bundle of $M(r, n)$ (see Lemma 3.6), hence $Z_{n}$ is the index of the Dirac operator, rather than the Dolbeault operator. Also, the factor makes the symmetry of the partition function nicer, as we see in Lemma 4.3.

Moreover, it is clear that this $K$-theoretic partition function converges to the homological partition function studied in [13, 14] as $\boldsymbol{\beta} \rightarrow 0$. 


\section{Blowup Equation AND a main Result}

2.1. Correlation functions on blowup. Let $\widehat{\mathbb{P}}^{2}$ be the blowup of $\mathbb{P}^{2}$ at $[1: 0: 0]$. Let $p: \widehat{\mathbb{P}}^{2} \rightarrow \mathbb{P}^{2}$ denote the projection. Let $C$ be the exceptional divisor, $\mathcal{O}(C)$ the corresponding line bundle, and $\mathcal{O}(m C)$ its $m$ th power.

Let $\widehat{M}(r, k, \widehat{n})$ be the framed moduli space of torsion free sheaves $(E, \Phi)$ on $\widehat{\mathbb{P}}^{2}$ with rank $r,\left\langle c_{1}(E),[C]\right\rangle=-k$ and $\left\langle\Delta(E),\left[\widehat{\mathbb{P}}^{2}\right]\right\rangle=\widehat{n}$ where $\Delta(E)=c_{2}(E)-\frac{r-1}{2 r} c_{1}(E)^{2}$. This is also nonsingular of dimension $2 \widehat{n} r$. (Remark that $\widehat{n}$ may not be integer in general.) By tensoring a line bundle if necessary, we assume $0 \leq k<r$ hereafter.

By [14, Theorem 3.3] there is a projective morphism defined by

$$
\begin{aligned}
\widehat{\pi}: \widehat{M}(r, k, \widehat{n}) & \rightarrow M_{0}(r, n) \\
(E, \Phi) & \mapsto\left(\left(\left(p_{*} E\right)^{\vee \vee}, \Phi\right), \operatorname{Supp}\left(p_{*} E^{\vee \vee} / p_{*} E\right)+\operatorname{Supp}\left(R^{1} p_{*} E\right)\right),
\end{aligned}
$$

where $n=\widehat{n}-\frac{k(r-k)}{2 r}$.

Let $(\mathcal{E}, \Phi)$ be the universal family on $\widehat{\mathbb{P}}^{2} \times \widehat{M}(r, k, \widehat{n})$. It has a natural $\widetilde{T}$-structure from the construction. As $C$ is $\mathbb{C}^{*} \times \mathbb{C}^{*}$-invariant, its fundamental class $[C]$ defines a class in the equivariant homology group. We define an equivariant $\mathbb{Q}$-divisor $\mu(C)$ on $\widehat{M}(r, k, \widehat{n})$ by

$$
\mu(C) \stackrel{\text { def. }}{=} p_{2 *}(\Delta(\mathcal{E}) \cap[C \times \widehat{M}(r, k, \widehat{n})]) \in A_{2 \widehat{T} r-1}^{\widetilde{T}}(\widehat{M}(r, k, \widehat{n}))_{\mathbb{Q}},
$$

where $p_{2}: \widehat{\mathbb{P}}^{2} \times \widehat{M}(r, k, \widehat{n}) \rightarrow \widehat{M}(r, k, \widehat{n})$ is the projection to the second factor. As customary, we denote the above as the 'slant product' $\Delta(\mathcal{E}) /[C]$. In the ordinary nonequivariant situation, it is known that $\mu(C)$ comes from the determinant line bundle

$$
\operatorname{det}\left(R^{\bullet} p_{2 *}\left(\mathcal{E}_{\mid C} \otimes p_{1}^{*} \mathcal{O}_{C}(-1)\right)\right) .
$$

(See [8, §1] for more detail). This construction works in our equivariant setting, so $\mu(C)$ comes from an equivariant $\mathbb{Q}$-line bundle. We will identify $\mu(C)$ as the latter element hereafter, but this does not make any trouble as $\left.\operatorname{Pic}^{\widetilde{T}}(\widehat{M}(r, k, \widehat{n})) \cong A_{2 \widehat{n} r-1}^{\widetilde{M}}(r, k, \widehat{n})\right)$ (see [19, Lemma 1.3] or [4, Theorem 1]).

We want to define the $K$-theoretic direct image $\widehat{\pi}_{*}(\mathcal{O}(d \mu(C)))$ of the $\widetilde{T}$-equivariant $\mathbb{Q}$-line bundle $\mu(C)$. For this purpose, we compute $\operatorname{det} \mathcal{E}$ for the universal family $(\mathcal{E}, \Phi)$ on $\widehat{\mathbb{P}}^{2} \times$ $\widehat{M}(r, k, \widehat{n})$. Since $h^{1}\left(\widehat{\mathbb{P}}^{2}\right)=0$, $\operatorname{det} \mathcal{E} \cong L \otimes \mathcal{O}_{\widehat{\mathbb{P}}^{2}}(k C)$ for a $\widetilde{T}$-line bundle $L$ on $\widehat{M}(r, k, \widehat{n})$. Since $\mathcal{E}_{\mid \ell_{\infty} \times \widehat{M}(r, k, \widehat{n})} \cong \bigoplus_{\alpha=1}^{r} \mathcal{O}_{\ell_{\infty} \times \widehat{M}(r, k, \widehat{n})} e_{\alpha}$ as $\widetilde{T}$-sheaves, we have $L \cong \mathcal{O}_{\widehat{M}(r, k, \widehat{n})} \prod_{\alpha} e_{\alpha}$. Hence $c_{1}(\mathcal{E})=\sum a_{\alpha}+k C$, therefore we have

$$
\begin{aligned}
\mu(C) & =\left(c_{2}(\mathcal{E})-\frac{r-1}{2 r}\left(\sum_{\alpha} a_{\alpha}+k C\right)^{2}\right) /[C] \\
& =c_{2}(\mathcal{E}) /[C]+\lambda,
\end{aligned}
$$

where $\lambda=\frac{r-1}{2 r}\left(2 k \sum_{\alpha} a_{\alpha}+k^{2}\left(\varepsilon_{1}+\varepsilon_{2}\right)\right) \in H^{2}(B \widetilde{T}, \mathbb{Q})$. Here we have used $1 /[C]=0, C /[C]=$ $-1, C^{2} /[C]=-\left(\varepsilon_{1}+\varepsilon_{2}\right)$ (cf. [13. Proof of Lemma 5.8]).

Now we define the $K$-theoretic direct image as follows:

\section{Definition 2.1.}

$$
\widehat{\pi}_{*}(\mathcal{O}(d \mu(C))) \stackrel{\text { def. }}{=} \widehat{\pi}_{*}\left(\mathcal{O}\left(d c_{2}(\mathcal{E}) /[C]\right)\right) \otimes \mathcal{O}_{M_{0}(r, n)}(d \lambda) \in K^{\widetilde{T}}\left(M_{0}(r, n)\right) \otimes_{R(\widetilde{T})} \mathcal{R}^{\prime},
$$


where $\mathcal{R}^{\prime}=\mathbb{Q}\left[t_{1}^{1 / r}, t_{2}^{1 / r}, e_{1}^{1 / r}, \ldots, e_{r}^{1 / r}\right]$.

Note that $\widehat{\pi}_{*}(\mathcal{O}(d \mu(C)))$ is in $K^{\widetilde{T}}\left(M_{0}(r, n)\right)$ if $k$ is 0 or $r$, or $d=0$.

We now define correlation functions on blowup:

$$
\widehat{Z}_{k, d}^{\text {inst }}\left(\varepsilon_{1}, \varepsilon_{2}, \vec{a} ; \mathfrak{q}, \boldsymbol{\beta}\right) \stackrel{\text { def. }}{=} \sum_{\widehat{n}}\left(\mathfrak{q} \boldsymbol{\beta}^{2 r} e^{-r \boldsymbol{\beta}\left(\varepsilon_{1}+\varepsilon_{2}\right) / 2}\right)^{\widehat{n}}\left(\iota_{0 *}\right)^{-1}\left(\widehat{\pi}_{*}(\mathcal{O}(d \mu(C)))\right) .
$$

2.2. Correlation functions via the partition function. As in $\$ 1.2$ we can use Atiyah-Bott Lefschetz fixed points formula to get another description of $\widehat{Z}_{k, d}^{\text {inst }}$. The necessary computation for the weights of tangent spaces and divisor $\mu(C)$ at fixed points was already done in homological version [13, $§ 3, \S 6]$. So we only state the answer:

$$
\begin{aligned}
& \widehat{Z}_{k, d}^{\text {inst }}\left(\varepsilon_{1}, \varepsilon_{2}, \vec{a} ; \mathfrak{q}, \boldsymbol{\beta}\right)=\sum_{\{\vec{k}\}=-k / r} \frac{\left(e^{\boldsymbol{\beta}\left(\varepsilon_{1}+\varepsilon_{2}\right)(d-r / 2)} \mathfrak{q} \boldsymbol{\beta}^{2 r}\right)^{(\vec{k}, \vec{k}) / 2} e^{\boldsymbol{\beta}(\vec{k}, \vec{a}) d}}{\prod_{\vec{\alpha} \in \Delta} l_{\vec{\alpha}}^{\vec{k}}\left(\varepsilon_{1}, \varepsilon_{2}, \vec{a}\right)} \times \\
& Z^{\text {inst }}\left(\varepsilon_{1}, \varepsilon_{2}-\varepsilon_{1}, \vec{a}+\varepsilon_{1} \vec{k} ; e^{\boldsymbol{\beta} \varepsilon_{1}(d-r / 2)} \mathfrak{q}, \boldsymbol{\beta}\right) Z^{\text {inst }}\left(\varepsilon_{1}-\varepsilon_{2}, \varepsilon_{2}, \vec{a}+\varepsilon_{2} \vec{k} ; e^{\boldsymbol{\beta} \varepsilon_{2}(d-r / 2)} \mathfrak{q}, \boldsymbol{\beta}\right) .
\end{aligned}
$$

We need explanations of several notations. The vector $\vec{a}$ is considered as an element of the Cartan subalgebra $\mathfrak{h}$ of $\mathfrak{s l}_{r}$ by imposing the condition $\sum_{\alpha} a_{\alpha}=0$. Then $\Delta$ is the set of roots of $\mathfrak{s l}_{r}$. The vector $\vec{k}$ runs over the coweight lattice $P=\left\{\vec{k}=\left(k_{1}, \ldots, k_{r}\right) \in \mathbb{Q}^{r} \mid \sum k_{\alpha}=0, \exists k \in\right.$ $\left.\mathbb{Z} \forall \alpha k_{\alpha} \equiv-k / r \bmod \mathbb{Z}\right\}$ with the constraint $\{\vec{k}\} \stackrel{\text { def. }}{=} k_{\alpha}(\bmod \mathbb{Z})=-k / r$. Finally we set

$$
l_{\vec{\alpha}}\left(\varepsilon_{1}, \varepsilon_{2}, \vec{a}\right)= \begin{cases}\prod_{\substack{i, j \geq 0 \\ i+j \leq-\langle\vec{k}, \vec{\alpha}\rangle-1}}^{i}\left(1-e^{\boldsymbol{\beta}\left(i \varepsilon_{1}+j \varepsilon_{2}-\langle\vec{a}, \vec{\alpha}\rangle\right)}\right) & \text { if }\langle\vec{k}, \vec{\alpha}\rangle<0, \\ \prod_{\substack{i, j \geq 0 \\ i+j \leq\langle\vec{k}, \vec{\alpha}\rangle-2}}\left(1-e^{\boldsymbol{\beta}\left(-(i+1) \varepsilon_{1}-(j+1) \varepsilon_{2}-\langle\vec{a}, \vec{\alpha}\rangle\right)}\right) & \text { if }\langle\vec{k}, \vec{\alpha}\rangle>1, \\ 1 & \text { otherwise }\end{cases}
$$

for a root $\vec{\alpha} \in \Delta$.

2.3. Main results. We can now state our main results:

Theorem 2.4. $(1)(d=0$ case $)$

$$
\widehat{Z}_{k, 0}^{\text {inst }}\left(\varepsilon_{1}, \varepsilon_{2}, \vec{a} ; \mathfrak{q}, \boldsymbol{\beta}\right)=\left(\mathfrak{q} \boldsymbol{\beta}^{2 r} e^{-r \boldsymbol{\beta}\left(\varepsilon_{1}+\varepsilon_{2}\right) / 2}\right)^{\frac{k(r-k)}{2 r}} Z^{\text {inst }}\left(\varepsilon_{1}, \varepsilon_{2}, \vec{a} ; \mathfrak{q}, \boldsymbol{\beta}\right) .
$$

$(2)(0<d<r$ case $)$

$$
\widehat{Z}_{k, d}^{\text {inst }}\left(\varepsilon_{1}, \varepsilon_{2}, \vec{a} ; \mathfrak{q}, \boldsymbol{\beta}\right)= \begin{cases}Z^{\text {inst }}\left(\varepsilon_{1}, \varepsilon_{2}, \vec{a} ; \mathfrak{q}, \boldsymbol{\beta}\right) & \text { for } k=0, \\ 0 & \text { for } 0<k<r .\end{cases}
$$

$(3)(d=r$ case $)$

$$
\widehat{Z}_{k, r}^{\text {inst }}\left(\varepsilon_{1}, \varepsilon_{2}, \vec{a} ; \mathfrak{q}, \boldsymbol{\beta}\right)=(-1)^{k(r-k)}\left(t_{1} t_{2}\right)^{k(r-k) / 2}\left(\mathfrak{q} \boldsymbol{\beta}^{2 r} e^{-r \boldsymbol{\beta}\left(\varepsilon_{1}+\varepsilon_{2}\right) / 2}\right)^{\frac{k(r-k)}{2 r}} Z^{\text {inst }}\left(\varepsilon_{1}, \varepsilon_{2}, \vec{a} ; \mathfrak{q}, \boldsymbol{\beta}\right)
$$

For the homological partition function, similar formulas were obtained ([13, 6.12], [14, §5.1]). The proof was the same as that of first several coefficients of the blowup formula for Donaldson invariants. It essentially follows from the dimension counting argument. We hope that the above formulas can be also considered as blowup formulas in low degree for the $K$-theoretic equivariant Donaldson invariants, whose existence is still conjectural. 
Combining Theorem 2.4 with (2.2), we get functional equations satisfied by $Z^{\text {inst }}$. These equations, which we call blowup equations, are powerful, and we have several consequences as we will see in later sections. We give the first application now:

Corollary 2.8. Let $Z_{n} \equiv Z_{n}\left(\varepsilon_{1}, \varepsilon_{2}, \vec{a} ; \boldsymbol{\beta}\right)$ be the coefficient of $\mathfrak{q}^{n}$ in $Z^{\text {inst }}\left(\varepsilon_{1}, \varepsilon_{2}, \vec{a} ; \mathfrak{q}, \boldsymbol{\beta}\right)$ as in (1.3). Then $Z_{n}$ is determined from $Z_{0}=1$ inductively by the blowup equations from Theorem 2.4 with $k=0, d=0,1,2$.

The proof is contained in that of Theorem 4.4

\section{VANISHING THEOREM}

We will prove Theorem 2.4 in this section. The crucial result here is the vanishing theorem of higher direct image sheaves (see Proposition [3.4). A reader who has interests only in applications of equations in Theorem 2.4 can safely skip this section.

3.1. It is known that $M_{0}(r, n)$ is a normal variety by [3]. Since $\pi: M(r, n) \rightarrow M_{0}(r, n)$ is birational, $\pi_{*}\left(\mathcal{O}_{M(r, n)}\right)=\mathcal{O}_{M_{0}(r, n)}$. Since $M(r, n)$ is a holomorphic symplectic manifold, $K_{M(r, n)} \cong$ $\mathcal{O}_{M(r, n)}$ as sheaves (see Lemma 3.6 for a different proof). By the Grauert-Riemenschneider vanishing theorem, the higher direct image sheaves vanish, i.e., $R^{i} \pi_{*}\left(\mathcal{O}_{M(r, n)}\right)=0$ for $i>0$. Hence we have the following.

Lemma 3.1. We have the following equality in the equivariant $K$-group $K^{\widetilde{T}}\left(M_{0}(r, n)\right)$ :

$$
\pi_{*}\left(\mathcal{O}_{M(r, n)}\right)=\mathcal{O}_{M_{0}(r, n)} .
$$

Theorem 2.4 is equivalent to the following:

Proposition 3.2. We have the following equalities in $K^{\widetilde{T}}\left(M_{0}(r, n)\right) \otimes_{R(\widetilde{T})} \mathcal{R}$ :

(2) If $0<d<r$, then

$$
\widehat{\pi}_{*}\left(\mathcal{O}_{\widehat{M}(r, k, \widehat{n})}\right)=\mathcal{O}_{M_{0}(r, n)}
$$

$$
\begin{gathered}
\widehat{\pi}_{*}\left(\mathcal{O}_{\widehat{M}(r, k, \widehat{n})}(d \mu(C))\right)= \begin{cases}\mathcal{O}_{M_{0}(r, n)}, & k=0 \\
0, & 0<k<r .\end{cases} \\
\widehat{\pi}_{*}\left(\mathcal{O}_{\widehat{M}(r, k, \widehat{n})}(r \mu(C))\right)=(-1)^{k(r-k)}\left(t_{1} t_{2}\right)^{k(r-k) / 2} \mathcal{O}_{M_{0}(r, n)} .
\end{gathered}
$$

The equation for the case where $0 \leq d<r$ is a consequence of the following two propositions.

Proposition 3.3. In the category of the equivariant coherent sheaves $\operatorname{Coh}_{\widetilde{T}}\left(M_{0}(r, n)\right)$, we have the following equalities:

(1)

(2) If $d \geq 1$, then

$$
\widehat{\pi}_{*}\left(\mathcal{O}_{\widehat{M}(r, k, \widehat{n})}\right)=\pi_{*}\left(\mathcal{O}_{M(r, n)}\right)
$$

$$
\widehat{\pi}_{*}\left(\mathcal{O}_{\widehat{M}(r, k, \widehat{n})}(d \mu(C))\right)= \begin{cases}\mathcal{O}_{M_{0}(r, n)}, & k=0 \\ 0, & 0<k<r\end{cases}
$$

\section{Proposition 3.4.}

$$
R^{i} \widehat{\pi}_{*}\left(\mathcal{O}_{\widehat{M}(r, k, \widehat{n})}(d \mu(C))\right)=0
$$

for $i>0$ and $d<r$. 
Proof of Proposition 3.3. We start with the proof of (1). We note that $\widehat{\pi}$ is a Grassmannian bundle over $M_{0}^{\mathrm{reg}}(r, n)$ with a fiber $G r(r, k)$. In fact, the inverse image of $(E, \Phi) \in M_{0}^{\mathrm{reg}}(r, n)$ under $\widehat{\pi}$ consists of sheaves $E^{\prime}$, which fit in an exact sequence

$$
0 \rightarrow E^{\prime}(-C) \rightarrow p^{*} E \rightarrow \mathcal{O}_{C}^{\oplus r-k} \rightarrow 0 \text {. }
$$

Thus $\mathcal{O}_{M_{0}(r, n)} \rightarrow \widehat{\pi}_{*}\left(\mathcal{O}_{\widehat{M}(r, k, \widehat{n})}\right)$ is an isomorphism on $M_{0}^{\mathrm{reg}}(r, n)$. Hence $\operatorname{Spec}\left(\widehat{\pi}_{*}\left(\mathcal{O}_{\widehat{M}(r, k, \widehat{n})}\right)\right) \rightarrow$ $M_{0}(r, n)$ is finite and birational. Since $M_{0}(r, n)$ is normal, the assertion holds.

We next prove (2). Assume that $k=0$. Then the Poincaré dual of $\mu(C)$ is the closed subset of $\widehat{M}(r, 0, \widehat{n})$ (see [2]):

$$
\left\{(E, \Phi) \in \widehat{M}(r, 0, \widehat{n}) \mid E_{\mid C} ¥ \mathcal{O}_{C}^{\oplus r}\right\} .
$$

Hence we have a $\widetilde{T}$-equivariant homomorphism $\mathcal{O}_{\widehat{M}(r, 0, \widehat{n})} \rightarrow \mathcal{O}_{\widehat{M}(r, 0, \widehat{n})}(\mu(C))$, which induces $\widetilde{T}$-equivariant inclusions $\mathcal{O}_{\widehat{M}(r, 0, \widehat{n})} \subset \mathcal{O}_{\widehat{M}(r, 0, \widehat{n})}(d \mu(C))$ for $d \geq 1$. By taking the direct images, we have a $\widetilde{T}$-equivariant inclusion

$$
\mathcal{O}_{M_{0}(r, n)}=\widehat{\pi}_{*}\left(\mathcal{O}_{\widehat{M}(r, 0, \widehat{n})}\right) \subset \widehat{\pi}_{*}\left(\mathcal{O}_{\widehat{M}(r, 0, \widehat{n})}(d \mu(C))\right) .
$$

We note that $\mathcal{O}_{M_{0}^{\text {reg }}(r, n)} \rightarrow \widehat{\pi}_{*}\left(\mathcal{O}_{\widehat{M}(r, 0, \widehat{n})}(d \mu(C))\right)_{\mid M_{0}^{\text {reg }}(r, n)}$ is an isomorphism. Since $M_{0}(r, n)$ is normal and $\operatorname{dim}\left(M_{0}(r, n) \backslash M_{0}^{\mathrm{reg}}(r, n)\right) \leq \operatorname{dim} M_{0}(r, n)-2$, the torsion freeness of $\widehat{\pi}_{*}\left(\mathcal{O}_{\widehat{M}(r, 0, \widehat{n})}(d \mu(C))\right)$ implies that $\widehat{\pi}_{*}\left(\mathcal{O}_{\widehat{M}(r, 0, \widehat{n})}(d \mu(C))\right)=\mathcal{O}_{M_{0}(r, n)}$ as a coherent sheaf. Then (3.5) implies that

$$
\widehat{\pi}_{*}\left(\mathcal{O}_{\widehat{M}(r, 0, \widehat{n})}(d \mu(C))\right) \cong \widehat{\pi}_{*}\left(\mathcal{O}_{\widehat{M}(r, 0, \widehat{n})}\right) \cong \mathcal{O}_{M_{0}(r, n)}
$$

as $\widetilde{T}$-equivariant sheaves. Thus (2) holds for $k=0$. We next assume that $0<k<r$. As we will see in Proposition 3.7. $-\mu(C)$ is $\widehat{\pi}$-big. Since $\widehat{\pi}$ is a $\operatorname{Gr}(r, k)$-bundle over $M_{0}^{\text {reg }}(r, n)$ with $\operatorname{dim} G r(r, k)=k(r-k)>0, \widehat{\pi}_{*}\left(\mathcal{O}_{\widehat{M}(r, 0, \widehat{n})}(d \mu(C))\right)_{\mid M_{0}^{\mathrm{reg}}(r, n)}=0$. Since $\widehat{\pi}_{*}\left(\mathcal{O}_{\widehat{M}(r, 0, \widehat{n})}(d \mu(C))\right)$ is torsion free, (2) holds also in these cases.

Proof of Proposition 3.4. We note that we do not need the $\widetilde{T}$-structure to prove the claim. So we forget the $\widetilde{T}$-action. In order to apply the Kawamata-Viehweg vanishing theorem to the $\mathbb{Q}$-line bundle $\mu(C)$, we first compute the canonical line bundle of $\widehat{M}(r, k, \widehat{n})$. For a later use, we compute it as a $\widetilde{T}$-equivariant sheaf.

Lemma 3.6. We have the following equalities:

$$
\begin{aligned}
K_{M(r, n)} & =\mathcal{O}_{M(r, n)}\left(r \mu\left(K_{\mathbb{P}^{2}}+2 \ell_{\infty}\right)\right)=\left(t_{1} t_{2}\right)^{-r n} \mathcal{O}_{M(r, n)} \in \operatorname{Pic}^{\widetilde{T}}(M(r, n)) \otimes \mathbb{Q}, \\
K_{\widehat{M}(r, k, \widehat{n})} & =\mathcal{O}_{\widehat{M}(r, k, \widehat{n})}\left(r \mu\left(K_{\widehat{\mathbb{P}} 2}+2 \ell_{\infty}\right)\right)=\left(t_{1} t_{2}\right)^{-r \widehat{n}} \mathcal{O}_{\widehat{M}(r, k, \widehat{n})}(r \mu(C)) \in \operatorname{Pic}^{\widetilde{T}}(\widehat{M}(r, k, \widehat{n})) \otimes \mathbb{Q} .
\end{aligned}
$$

Proof. We only compute $K_{\widehat{M}(r, k, \widehat{n})}$. The computation of $K_{M(r, n)}$ is similar and simpler. Let $(\mathcal{E}, \Phi)$ be the universal family on $\widehat{\mathbb{P}}^{2} \times \widehat{M}(r, k, \widehat{n})$ and $p_{2}: \widehat{\mathbb{P}}^{2} \times \widehat{M}(r, k, \widehat{n}) \rightarrow \widehat{M}(r, k, \widehat{n})$ be the projection. Note that $T_{\widehat{M}(r, k, \widehat{n})} \cong \operatorname{Ext}_{p_{2}}^{1}\left(\mathcal{E}, \mathcal{E}\left(-\ell_{\infty}\right)\right)$. Using the (equivariant) GrothendieckRiemann-Roch theorem, we see that

$$
\begin{aligned}
& c_{1}\left(K_{\widehat{M}(r, k, \widehat{n})}\right) \\
= & c_{1}\left(\mathbf{R} p_{2 *}\left(\mathcal{E}^{\vee} \otimes \mathcal{E}\left(-\ell_{\infty}\right)\right)\right) \\
= & {\left[p_{2 *}\left(\operatorname{ch}\left(\mathcal{E}^{\vee} \otimes \mathcal{E}\right) e^{-\ell_{\infty}} \operatorname{Todd}_{\widehat{\mathbb{P}}^{2}}\right)\right]_{1} } \\
= & {\left[p_{2 *}\left(\left(r^{2}-r \Delta(\mathcal{E})+\operatorname{ch}_{4}\left(\mathcal{E}^{\vee} \otimes \mathcal{E}\right)+\cdots\right)\left(1-\frac{2 \ell_{\infty}+K_{\widehat{\mathbb{P}}^{2}}}{2}+\cdots\right)\right)\right]_{1} } \\
= & r \Delta(\mathcal{E}) /\left(K_{\widehat{\mathbb{P}}^{2}}+2 \ell_{\infty}\right)=r \mu\left(K_{\widehat{\mathbb{P}}^{2}}+2 \ell_{\infty}\right),
\end{aligned}
$$


where $[\ldots]_{1}$ means the codimension 1 component in the equivariant Chow group $A_{2 \widehat{n} r-1}^{\widetilde{T}}(\widehat{M}(r, k, \widehat{n}))_{\mathbb{Q}}$. Since $\operatorname{Pic}^{\widetilde{T}}(\widehat{M}(r, k, \widehat{n})) \cong A_{2 \widehat{T} r-1}^{\widetilde{T}}(\widehat{M}(r, k, \widehat{n}))$ (see [19, Lemma 1.3] or [4], Theorem 1]), $K_{\widehat{M}(r, k, \widehat{n})}=$ $\mathcal{O}_{\widehat{M}(r, k, \widehat{n})}\left(r \mu\left(K_{\widehat{\mathbb{P}}^{2}}+2 \ell_{\infty}\right)\right) \in \operatorname{Pic}^{\widetilde{T}}(\widehat{M}(r, k, \widehat{n})) \otimes \mathbb{Q}$. Since $\mathcal{E}_{\mid \ell_{\infty} \times \widehat{M}(r, k, \widehat{n})} \cong \bigoplus_{\alpha=1}^{r} \mathcal{O}_{\ell_{\infty} \times \widehat{M}(r, k, \widehat{n})} e_{\alpha}$ as $\widetilde{T}$-sheaves, we have $p_{2 *}\left(\Delta\left(\mathcal{E}_{\mid \ell_{\infty} \times \widehat{M}(r, k, \widehat{n})}\right)\right)=0$ in $A_{\widetilde{T}}^{1}(\widehat{M}(r, k, \widehat{n}))_{\mathbb{Q}}$. Therefore $\mathcal{O}_{\widehat{M}(r, k, n)}\left(\mu\left(\ell_{\infty}\right)\right) \cong$ $\mathcal{O}_{\widehat{M}(r, k, n)}$ as a $\widetilde{T}$-line bundle. Since $K_{\mathbb{P}^{2}} \cong\left(t_{1} t_{2}\right)^{-1} \mathcal{O}_{\mathbb{P}^{2}}\left(-3 \ell_{\infty}\right)$ and $K_{\widehat{\mathbb{P}}^{2}} \cong p^{*}\left(K_{\mathbb{P}^{2}}\right)(C)$, we get

$$
K_{\widehat{M}(r, k, \widehat{n})}=\left(t_{1} t_{2}\right)^{-r \widehat{n}} \mathcal{O}_{\widehat{M}(r, k, \widehat{n})}(r \mu(C)) \in \operatorname{Pic}^{\widetilde{T}}(\widehat{M}(r, k, \widehat{n})) \otimes \mathbb{Q},
$$

where we used $\Delta(\mathcal{E}) /\left[\widehat{\mathbb{P}}^{2}\right]=\widehat{n}$.

By this lemma, $\mathcal{O}_{\widehat{M}(r, k, \widehat{n})}(d \mu(C))=K_{\widehat{M}(r, k, \widehat{n})}((d-r) \mu(C)) \in \operatorname{Pic}^{\widetilde{T}}(\widehat{M}(r, k, \widehat{n})) \otimes \mathbb{Q}$. By the following proposition, $-\mu(C)$ on $\widehat{M}(r, k, \widehat{n})$ is $\widehat{\pi}$-nef and $\widehat{\pi}$-big. Then the relative version of the Kawamata-Viehweg vanishing theorem [7, Theorem 1-2-3] implies that

$$
R^{i} \widehat{\pi}_{*}\left(\mathcal{O}_{\widehat{M}(r, k, \widehat{n})}(d \mu(C))\right) \cong R^{i} \widehat{\pi}_{*}\left(K_{\widehat{M}(r, k, \widehat{n})}((d-r) \mu(C)+D)\right)=0
$$

for $d<r$, where $D=0$ in $A_{2 \widehat{n} r-1}(\widehat{M}(r, k, \widehat{n})) \otimes \mathbb{Q}$. Thus Proposition 3.4 holds.

Proposition 3.7. The $\mathbb{Q}$-divisor $-\mu(C)$ on $\widehat{M}(r, k, \widehat{n})$ is $\widehat{\pi}$-nef and $\widehat{\pi}$-big.

Before proving this proposition, we shall treat the remaining case (3) $d=r$ and finish the proof of Proposition 3.2. Since $\widehat{\pi}_{*}\left(\mathcal{O}_{\widehat{M}(r, k, \widehat{n})}\right)=\mathcal{O}_{M_{0}(r, n)}$ in $K^{\widetilde{T}}\left(M_{0}(r, n)\right)$, the GrothendieckSerre duality implies that $\widehat{\pi}_{*}\left(K_{\widehat{M}(r, k, \widehat{n})}\right)=(-1)^{k(r-k)} K_{M_{0}(r, n)}=(-1)^{k(r-k)} \pi_{*}\left(K_{M(r, n)}\right)$ in $K^{\widetilde{T}}\left(M_{0}(r, n)\right)$. By using Lemma 3.6, we get (3).

The proof of Proposition 3.7 occupies the rest of this section. Let us briefly sketch the idea of the proof. Let $M\left(r,-H, n^{\prime}\right)$ be the moduli space of $H$-stable sheaves $E$ on $\mathbb{P}^{2}$ with $c_{1}(E)=c_{1}, \Delta(E)=n^{\prime}=n+(r-1)(2 r-1) / r$. The first step of the proof is to construct an embedding $M(r, n) \rightarrow M\left(r,-H, n^{\prime}\right)$ by using idea in [11. Next we show that there is an induced commutative diagram

$$
\begin{array}{ccc}
M(r, n) & \hookrightarrow & M\left(r,-H, n^{\prime}\right) \\
\pi \downarrow & & \downarrow \pi^{\prime} \\
M_{0}(r, n) & \rightarrow & M_{0}\left(r,-H, n^{\prime}\right),
\end{array}
$$

where $M_{0}\left(r,-H, n^{\prime}\right)$ is the Uhlenbeck compactification of the open subset consisting of locally free sheaves in $M\left(r,-H, n^{\prime}\right)$, and $\pi^{\prime}$ is the morphism defined exactly as $\pi$. Similarly we can make a commutative diagram on blowup:

$$
\begin{array}{ccc}
\widehat{M}(r, k, \widehat{n}) & \hookrightarrow & \widehat{M}\left(r, k C-H, \widehat{n}^{\prime}\right) \\
\widehat{\pi} \downarrow & & \downarrow \widehat{\pi}^{\prime} \\
M_{0}(r, n) & \rightarrow & M_{0}\left(r,-H, n^{\prime}\right),
\end{array}
$$

where $\widehat{M}\left(r, k C-H, \widehat{n}^{\prime}\right)$ is the moduli space of $(H-\varepsilon C)$-stable sheaves on $\widehat{\mathbb{P}}^{2}$ with $\widehat{n}^{\prime}-\widehat{n}=n^{\prime}-n$. From the diagram it is enough to show that $-\mu(C)$ is $\widehat{\pi}^{\prime}$-nef and $\widehat{\pi}^{\prime}$-big. But this assertion follows from the following known results: 1) The divisor $\mu(H-\varepsilon C)$ is nef and big as it gives a birational morphism $\widehat{M}\left(r, k C-H, \widehat{n}^{\prime}\right) \rightarrow \widehat{M}_{0}\left(r, k C-H, \widehat{n}^{\prime}\right)$ 9. 2) $\mu(H)$ is the pull-back of a $\mathbb{Q}$-Cartier divisor $\mathcal{H}$ on $M_{0}\left(r,-H, n^{\prime}\right)$ by the construction of $\widehat{\pi}^{\prime}$ in [14, Appendix F]. 
3.2. An embedding $M(r, n) \rightarrow M\left(r,-H, n^{\prime}\right)$. We construct an embedding $M(r, n) \rightarrow M\left(r,-H, n^{\prime}\right)$ using parabolic sheaves [12]. Our construction is inspired by [11]. We collect basic facts on parabolic sheaves in [12].

Definition 3.10. A parabolic sheaf $\left(E_{*}, \alpha_{*}\right)$ with respect to $\ell_{\infty}$ is a pair of a filtration

$$
E_{*}: E\left(-\ell_{\infty}\right) \subset E_{l} \subset \cdots \subset E_{2} \subset E_{1}=E
$$

on $\mathbb{P}^{2}$ and a sequence of rational numbers $0 \leq \alpha_{1}<\alpha_{2}<\cdots<\alpha_{l}<1$.

In this paper, we consider parabolic sheaves $\left(E_{*}, \alpha_{1}, \alpha_{2}\right)$ such that

$$
E_{*}: E\left(-\ell_{\infty}\right) \subset E^{\prime} \subset E
$$

and $E / E^{\prime}=\mathcal{O}_{\ell_{\infty}}(r-1)$ in the Grothendieck group $K\left(\ell_{\infty}\right)$.

Definition 3.12. A parabolic sheaf $\left(E_{*}, \alpha_{1}, \alpha_{2}\right)$ is $\mu$-semi-stable, if $E$ is torsion free and

$$
\begin{aligned}
& \frac{\operatorname{deg} G+\alpha_{1} \operatorname{rank}_{\ell_{\infty}}\left(G / G \cap E^{\prime}\right)+\alpha_{2} \operatorname{rank}_{\ell_{\infty}}\left(G \cap E^{\prime} / G\left(-\ell_{\infty}\right)\right)}{\operatorname{rank} G} \\
\leq & \frac{\operatorname{deg} E+\alpha_{1} \operatorname{rank}_{\ell_{\infty}}\left(E / E^{\prime}\right)+\alpha_{2} \operatorname{rank}_{\ell_{\infty}}\left(E^{\prime} / E\left(-\ell_{\infty}\right)\right)}{\operatorname{rank} E}
\end{aligned}
$$

for a saturated subsheaf $G \subset E$ with $0<\operatorname{rank} G<\operatorname{rank} E$, where deg denotes the degree with respect to $H$ and $\operatorname{rank}_{\ell_{\infty}}$ the rank on $\ell_{\infty}$. If the inequality is strict for all $G$, then we say $\left(E_{*}, \alpha_{1}, \alpha_{2}\right)$ is $\mu$-stable.

We set $\alpha=\alpha_{2}-\alpha_{1}$, then $0<\alpha<1$ and (3.13) is equivalent to the following condition:

$$
\frac{\operatorname{deg} G-\alpha \operatorname{rank}_{\ell_{\infty}}\left(G / G \cap E^{\prime}\right)}{\operatorname{rank} G} \leq \frac{\operatorname{deg} E-\alpha \operatorname{rank}_{\ell_{\infty}}\left(E / E^{\prime}\right)}{\operatorname{rank} E}
$$

for a saturated subsheaf $G \subset E$ with $0<\operatorname{rank} G<\operatorname{rank} E$. Therefore we define our parabolic sheaf as a filtration $E_{*}: E\left(-\ell_{\infty}\right) \subset E^{\prime} \subset E$ with a parameter $\alpha$, and we define the stability of $\left(E_{*}, \alpha\right)$ as the condition (3.14).

From now on, we fix the parameter $\alpha$ with $0<\alpha<r /(r-1)^{2}$. Thus our parabolic sheaf is the filtration $E_{*}: E\left(-\ell_{\infty}\right) \subset E^{\prime} \subset E$.

Definition 3.15. $\mathfrak{M}$ denotes the moduli space of $\mu$-stable parabolic sheaves $\left(E_{*}, \alpha\right)$ on $\mathbb{P}^{2}$ such that $\left(\operatorname{rank} E, c_{1}(E), \Delta(E)\right)=(r, 0, n)$ and $E / E^{\prime}=\mathcal{O}_{\ell_{\infty}}(r-1)$ in $K\left(\ell_{\infty}\right)$.

An easy computation shows that $\left(c_{1}\left(E^{\prime}\right), \Delta\left(E^{\prime}\right)\right)=\left(-H, n^{\prime}\right)$.

Lemma 3.16. Let $E_{*}: E\left(-\ell_{\infty}\right) \subset E^{\prime} \subset E$ be a parabolic sheaf with (rank $\left.E, c_{1}(E), \Delta(E)\right)=$ $(r, 0, n)$ and $E / E^{\prime}=\mathcal{O}_{\ell_{\infty}}(r-1)$.

(1) $E_{*}$ is $\mu$-stable if and only if $E$ is $\mu$-semi-stable and $\operatorname{rank}_{\ell_{\infty}}\left(G / G \cap E^{\prime}\right)=1$ for all saturated subsheaf $0 \neq G \subset E$ with $\operatorname{deg}(G)=0$.

(2) $E_{*}$ is $\mu$-stable if and only if $E^{\prime}$ is $\mu$-stable. Hence we have a morphism $\phi: \mathfrak{M} \rightarrow$ $M\left(r,-H, n^{\prime}\right)$. 
Proof. We first prove (1). Assume that $E_{*}$ is $\mu$-stable. If $E$ is not $\mu$-semi-stable, then there is a saturated subsheaf $G \subset E$ with $\operatorname{deg} G>0$. Then

$$
\begin{aligned}
& \frac{\operatorname{deg} E-\alpha \operatorname{rank}_{\ell_{\infty}}\left(E / E^{\prime}\right)}{\operatorname{rank} E}-\frac{\operatorname{deg} G-\alpha \operatorname{rank}_{\ell_{\infty}}\left(G / G \cap E^{\prime}\right)}{\operatorname{rank} G} \\
\leq & \frac{-1}{r-1}+\alpha\left(\frac{\operatorname{rank}_{\ell_{\infty}}\left(G / E^{\prime} \cap G\right)}{\operatorname{rank} G}-\frac{1}{r}\right) \\
\leq & \frac{-1}{r-1}+\alpha\left(1-\frac{1}{r}\right) \leq \frac{1}{r(r-1)}\left((r-1)^{2} \alpha-r\right)<0,
\end{aligned}
$$

which is a contradiction. Therefore $E$ is $\mu$-semi-stable. If $E$ is properly $\mu$-semi-stable, then there is a saturated subsheaf $G \subset E$ with $\operatorname{deg} G=0$. In this case, we must have $\operatorname{rank}_{\ell_{\infty}}\left(G / G \cap E^{\prime}\right) / \operatorname{rank} G>1 / r$. Since $G / G \cap E^{\prime} \subset E / E^{\prime}$ and $\operatorname{rank}_{\ell_{\infty}}\left(E / E^{\prime}\right)=1, \operatorname{rank}_{\ell_{\infty}}(G / G \cap$ $\left.E^{\prime}\right)=1$. We show the inverse direction. We assume that $E$ is $\mu$-stable. Then for a saturated subsheaf $G \subset E$, we have $\operatorname{deg} G<0$. Then we see that

$$
\begin{aligned}
& \frac{\operatorname{deg} E-\alpha \operatorname{rank}_{\ell_{\infty}}\left(E / E^{\prime}\right)}{\operatorname{rank} E}-\frac{\operatorname{deg} G-\alpha \operatorname{rank}_{\ell_{\infty}}\left(G / G \cap E^{\prime}\right)}{\operatorname{rank} G} \\
\geq & \frac{1}{r-1}+\alpha\left(\frac{\operatorname{rank}_{\ell_{\infty}}\left(G / E^{\prime} \cap G\right)}{\operatorname{rank} G}-\frac{1}{r}\right) \geq \frac{1}{r-1}-\frac{\alpha}{r}>0 .
\end{aligned}
$$

Thus $E_{*}$ is $\mu$-stable. If $E$ is properly $\mu$-semi-stable, we assume that $\operatorname{rank}_{\ell_{\infty}}\left(G / G \cap E^{\prime}\right)=1$ for all saturated subsheaf $G \subset E$ with $\operatorname{deg} G=0$. Then we also see that $E_{*}$ is $\mu$-stable.

We next prove (2). If $E^{\prime}$ is not $\mu$-stable, then there is a saturated subsheaf $G^{\prime} \subset E^{\prime}$ with $0<\operatorname{rank} G^{\prime}<\operatorname{rank} E^{\prime}$ and

$$
\frac{\operatorname{deg} G^{\prime}}{\operatorname{rank} G^{\prime}} \geq \frac{\operatorname{deg}\left(E^{\prime}\right)}{\operatorname{rank} E^{\prime}}=-\frac{1}{r}
$$

Therefore $\operatorname{deg} G^{\prime} \geq 0$. Since $E^{\prime} / G^{\prime}$ is torsion free, $G_{\mid \ell_{\infty}}^{\prime} \rightarrow E_{\mid \ell_{\infty}}^{\prime}$ is injective, and hence $G^{\prime} \cap$ $E^{\prime}\left(-\ell_{\infty}\right)=G^{\prime}\left(-\ell_{\infty}\right)$. We set $G:=\left(G^{\prime} \cap E\left(-\ell_{\infty}\right)\right)\left(\ell_{\infty}\right)$. Since $\left(G^{\prime} \cap E\left(-\ell_{\infty}\right)\right) \cap E^{\prime}\left(-\ell_{\infty}\right)=$ $G^{\prime} \cap E^{\prime}\left(-\ell_{\infty}\right)=G^{\prime}\left(-\ell_{\infty}\right)$, we get $G \cap E^{\prime}=G^{\prime}$. By $(1), E$ is $\mu$-semi-stable, therefore $\operatorname{deg} G \leq 0$. As $0 \leq \operatorname{deg} G^{\prime} \leq \operatorname{deg} G \leq 0$, we get $\operatorname{deg} G=\operatorname{deg} G^{\prime}=0$. Hence $\operatorname{rank}_{\ell_{\infty}}\left(G / G^{\prime}\right)=0$, which implies that $E\left(-\ell_{\infty}\right) \subset E^{\prime} \subset E$ is not $\mu$-semi-stable.

Conversely suppose $E^{\prime}$ is $\mu$-stable. Then for a subsheaf $G \subset E$, we have $\operatorname{deg} G \cap E^{\prime}<$ 0. As $\operatorname{deg} G=\operatorname{deg}\left(G \cap E^{\prime}\right)+\operatorname{rank}_{\ell_{\infty}}\left(G / G \cap E^{\prime}\right)$, we have $\operatorname{deg} G<0$ or $\operatorname{deg} G=0$ and $\operatorname{rank}\left(G / G \cap E^{\prime}\right)=1$. By $(1), E_{*}$ is $\mu$-stable.

Let $\mathfrak{M}^{\prime}$ be the open subscheme of $\mathfrak{M}$ such that

$$
\begin{aligned}
\mathfrak{M}^{\prime} & =\left\{E_{*} \in \mathfrak{M} \mid E / E^{\prime} \text { and } E^{\prime} / E\left(-\ell_{\infty}\right) \text { are semi-stable locally free sheaves on } \ell_{\infty}\right\} \\
& =\left\{E_{*} \in \mathfrak{M} \mid E / E^{\prime} \cong \mathcal{O}_{\ell_{\infty}}(r-1), E^{\prime} / E\left(-\ell_{\infty}\right) \cong \mathcal{O}_{\ell_{\infty}}(-1)^{\oplus(r-1)}\right\} .
\end{aligned}
$$

If $E\left(-\ell_{\infty}\right) \subset E^{\prime} \subset E$ belongs to $\mathfrak{M}^{\prime}$, then $E_{\mid \ell_{\infty}}$ is a locally free $\mathcal{O}_{\ell_{\infty}}$-module. Hence $E$ is locally free in a neighborhood of $\ell_{\infty}$, and hence $E^{\prime}=\operatorname{ker}\left(E \rightarrow \mathcal{O}_{\ell_{\infty}}(r-1)\right)$ is also locally free in a neighborhood of $\ell_{\infty}$.

Lemma 3.17. Let $E_{*}: E\left(-\ell_{\infty}\right) \subset E^{\prime} \subset E$ be a point of $\mathfrak{M}$.

(1) If $E_{*}$ belongs to $\mathfrak{M}^{\prime}$, then $E_{\mid \ell_{\infty}}^{\prime} \cong \mathcal{O}_{\ell_{\infty}}(r-2) \oplus \mathcal{O}_{\ell_{\infty}}(-1)^{\oplus(r-1)}$.

(2) If $E_{\mid \ell_{\infty}}^{\prime} \cong \mathcal{O}_{\ell_{\infty}}(r-2) \oplus \mathcal{O}_{\ell_{\infty}}(-1)^{\oplus(r-1)}$, then

$$
E=\operatorname{ker}\left(E^{\prime} \rightarrow \operatorname{Hom}\left(E^{\prime}, \mathcal{O}_{\ell_{\infty}}(-1)\right)^{\vee} \otimes \mathcal{O}_{\ell_{\infty}}(-1)\right) \otimes \mathcal{O}_{\mathbb{P}^{2}}\left(\ell_{\infty}\right) .
$$

In particular $E_{*} \in \mathfrak{M}$ is uniquely determined by $E^{\prime}$. Moreover $E_{*}$ belongs to $\mathfrak{M}^{\prime}$. 
Proof. (1) By the filtration $E^{\prime}\left(-\ell_{\infty}\right) \subset E\left(-\ell_{\infty}\right) \subset E^{\prime} \subset E$ induced by $E_{*}$, we have an exact sequence

$$
0 \rightarrow\left(E / E^{\prime}\right) \otimes \mathcal{O}_{\mathbb{P}^{2}}\left(-\ell_{\infty}\right) \rightarrow E_{\mid \ell_{\infty}}^{\prime} \rightarrow E^{\prime} / E\left(-\ell_{\infty}\right) \rightarrow 0 .
$$

Then this exact sequence splits and we get our claim.

(2) We set $L^{\prime}:=E^{\prime} / E\left(-\ell_{\infty}\right)$. Then $L^{\prime}$ is an $\mathcal{O}_{\ell_{\infty}}$-module of rank $r-1$ with $\operatorname{deg} L^{\prime}=-(r-1)$. Let $T$ be the torsion submodule of $L^{\prime}$ and

$$
0 \subset F_{1} \subset F_{2} \subset \cdots \subset F_{s}=L^{\prime} / T
$$

the Harder-Narasimhan filtration of $L^{\prime} / T$. We set $F_{i} / F_{i-1} \cong \mathcal{O}_{\ell_{\infty}}\left(a_{i}\right)^{\oplus n_{i}}$. Then $a_{1}>a_{2}>\cdots>$ $a_{s}$ with $\sum_{i=1}^{s} n_{i} a_{i}+\operatorname{deg}(T)=\operatorname{deg} L^{\prime}=-(r-1)$. Since we have a surjective homomorphism

$$
E_{\mid \ell_{\infty}}^{\prime} \rightarrow L^{\prime} \rightarrow \mathcal{O}_{\ell_{\infty}}\left(a_{s}\right)
$$

we get $a_{s} \geq-1$. Then we see that $T=0, s=1$ and $L^{\prime} \cong \mathcal{O}_{\ell_{\infty}}(-1)^{\oplus(r-1)}$. Moreover we have a commutative diagram

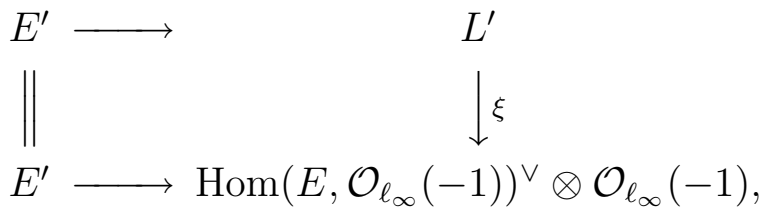

where $\xi$ is an isomorphism. Hence (3.18) holds. By the filtration $E^{\prime}\left(-\ell_{\infty}\right) \subset E\left(-\ell_{\infty}\right) \subset E^{\prime}$ induced by $E_{*}$, we see that $E / E^{\prime} \cong \mathcal{O}_{\ell_{\infty}}(r-1)$. Therefore $(2)$ holds.

We set

$$
S:=\left\{E^{\prime} \in M\left(r,-H, n^{\prime}\right) \mid E_{\mid \ell_{\infty}}^{\prime} \cong \mathcal{O}_{\ell_{\infty}}(r-2) \oplus \mathcal{O}_{\ell_{\infty}}(-1)^{\oplus(r-1)}\right\} .
$$

$S$ is a locally closed subscheme of $M\left(r,-H, n^{\prime}\right)$, where we use the reduced scheme structure on $S$. By Lemma 3.17 (1), we get $\phi\left(\mathfrak{M}^{\prime}\right) \subset S$.

Lemma 3.19. $\mathfrak{M}^{\prime} \rightarrow S$ is an isomorphism.

Proof. Let $\mathcal{E}^{\prime}$ be the universal family on $S \times \mathbb{P}^{2}$ and $q_{S}: S \times \mathbb{P}^{2} \rightarrow S$ the projection. By the definition of $S, \operatorname{Hom}\left(\mathcal{E}_{\mid\{s\} \times \ell_{\infty}}^{\prime}, \mathcal{O}_{\ell_{\infty}}(-1)\right) \cong \mathbb{C}^{\oplus(r-1)}$ for $s \in S$. Since $S$ is reduced, the base change theorem implies that $U:=\operatorname{Hom}_{q_{S}}\left(\mathcal{E}_{\mid S \times \ell_{\infty}}^{\prime}, \mathcal{O}_{S \times \ell_{\infty}}(-1)\right)$ is a locally free sheaf on $S$ and we have a family of homomorphisms

$$
f: \mathcal{E}_{\mid S \times \mathbb{P}^{2}}^{\prime} \rightarrow U^{\vee} \otimes \mathcal{O}_{\ell_{\infty}}(-1)
$$

We set $\mathcal{E}:=(\operatorname{ker} f)\left(\ell_{\infty}\right)$. Then we have a family of parabolic sheaves $\mathcal{E}\left(-\ell_{\infty}\right) \subset \mathcal{E}^{\prime} \subset \mathcal{E}$. They are $\mu$-stable by Lemma 3.16 (2). Thus we have a morphism $\psi: S \rightarrow \mathfrak{M}^{\prime}$. It is easy to see that $\phi \circ \psi$ is the identity. By Lemma 3.17 (2), we also see that $\psi \circ \phi$ is the identity. Therefore $\phi$ is an isomorphism.

Let $(\mathcal{E}, \Phi)$ be the universal family on $M(r, n) \times \mathbb{P}^{2}$. Then we have a surjective homomorphism

$$
\Psi: \mathcal{E} \rightarrow \mathcal{O}_{M(r, n) \times \ell_{\infty}}^{\oplus r} \stackrel{\phi}{\rightarrow} \mathcal{O}_{M(r, n) \times \ell_{\infty}}(r-1)
$$

where $\phi:=\left(z_{1}^{r-1}, z_{1}^{r-2} z_{2}, \ldots, z_{2}^{r-1}\right)$. We set $\mathcal{E}^{\prime}:=\operatorname{ker} \Psi$. Then we have a family of parabolic sheaves $\mathcal{E}\left(-\ell_{\infty}\right) \subset \mathcal{E}^{\prime} \subset \mathcal{E}$.

Lemma 3.20. $\mathcal{E}\left(-\ell_{\infty}\right) \subset \mathcal{E}^{\prime} \subset \mathcal{E}$ is a family of $\mu$-stable parabolic sheaves. 
Proof. For $s \in M(r, n)$, we set $E:=\mathcal{E}_{\mid\{s\} \times \mathbb{P}^{2}}$ and $E^{\prime}:=\mathcal{E}_{\mid\{s\} \times \mathbb{P}^{2}}^{\prime}$. Since $E_{\ell_{\infty}} \cong \mathcal{O}_{\ell_{\infty}}^{\oplus r}, E$ is $\mu$ semi-stable. Assume that there is a saturated proper subsheaf $G$ of $E$ with $\operatorname{deg} G=0$. Then, since $E / G$ is torsion free, $G_{\mid \ell_{\infty}}$ is a subsheaf of $E_{\mid \ell_{\infty}} \cong \mathcal{O}_{\ell_{\infty}}^{\oplus r}$. Hence $G_{\mid \ell_{\infty}}$ is a direct summand

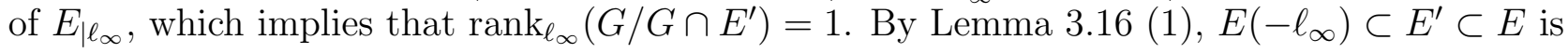
$\mu$-stable.

Hence we have a morphism $M(r, n) \rightarrow \mathfrak{M}$. Let $\mathfrak{M}^{\prime \prime}$ be the open subscheme of $\mathfrak{M}^{\prime}$ such that $E\left(-\ell_{\infty}\right) \subset E^{\prime} \subset E$ satisfies $E_{\mid \ell_{\infty}} \cong \mathcal{O}_{\ell_{\infty}}^{\oplus r}$.

Lemma 3.21. We have an isomorphism $M(r, n) \rightarrow \mathfrak{M}^{\prime \prime}$.

Proof. We construct the inverse map. For the family of parabolic sheaves $\mathcal{E}\left(-\ell_{\infty}\right) \subset \mathcal{E}^{\prime} \subset \mathcal{E}$ on $\mathfrak{M}^{\prime \prime} \times \mathbb{P}^{2}$, we see that $\mathcal{E} / \mathcal{E}^{\prime} \cong L \otimes \mathcal{O}_{\ell_{\infty}}(r-1)$ for a line bundle $L$ on $\mathfrak{M}^{\prime \prime}$. By taking the tensor product with $q_{\mathfrak{M}^{\prime \prime}}^{*}\left(L^{\vee}\right)$ to the family, we may assume that $\mathcal{E} / \mathcal{E}^{\prime} \cong \mathcal{O}_{\mathfrak{M}^{\prime \prime}} \otimes \mathcal{O}_{\ell_{\infty}}(r-1)$. We set $g: \mathcal{E} \rightarrow \mathcal{O}_{\mathfrak{M}^{\prime \prime}} \otimes \mathcal{O}_{\ell_{\infty}}(r-1)$. Since $\mathcal{E}_{\mid \ell_{\infty}}$ is a family of $\mathcal{O}_{\ell_{\infty}}^{\oplus r}, g$ induces an isomorphism $q_{\mathfrak{M}^{\prime \prime} *}\left(\mathcal{E}_{\mid \ell_{\infty}}\right) \rightarrow \mathcal{O}_{\mathfrak{M}^{\prime \prime}} \otimes H^{0}\left(\ell_{\infty}, \mathcal{O}_{\ell_{\infty}}(r-1)\right)$. Then we have a commutative diagram

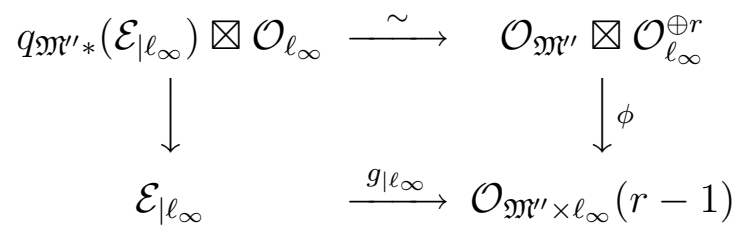

Since the left vertical map is an isomorphism, we can find a homomorphism $\Phi: \mathcal{E} \rightarrow \mathcal{O}_{\mathfrak{M}^{\prime \prime} \times \ell_{\infty}}^{\oplus r}$ such that $\phi \circ \Phi=g$.

By Lemma 3.19 and Lemma 3.21, we have a sequence of morphisms:

$$
M(r, n) \stackrel{\sim}{\rightarrow} \mathfrak{M}^{\prime \prime} \hookrightarrow \mathfrak{M}^{\prime} \stackrel{\sim}{\rightarrow} S \hookrightarrow M\left(r,-H, n^{\prime}\right) .
$$

Thus we obtain the following proposition.

Proposition 3.22. We have an immersion $\iota: M(r, n) \rightarrow M\left(r,-H, n^{\prime}\right)$.

3.3. Construction of $M_{0}(r, n) \rightarrow M_{0}\left(r,-H, n^{\prime}\right)$. Let $\pi^{\prime}: M\left(r,-H, n^{\prime}\right) \rightarrow M_{0}\left(r,-H, n^{\prime}\right)$ be the contraction map to the Uhlenbeck's compactification of the open subset consisting of locally free sheaves in $M\left(r,-H, n^{\prime}\right)$. Since $\pi^{\prime}$ is surjective, replacing $M_{0}\left(r,-H, n^{\prime}\right)$ by its normalization, we may assume that $M_{0}\left(r,-H, n^{\prime}\right)$ is normal. Then by Corollary A.2 $M_{0}\left(r,-H, n^{\prime}\right)$ is the quotient of the equivalence relation which defines the contraction to the Uhlenbeck compactification. We consider the composition $\varpi: M(r, n) \rightarrow M\left(r,-H, n^{\prime}\right) \rightarrow M_{0}\left(r,-H, n^{\prime}\right)$. Then

Lemma 3.23. $\varpi\left(\left(E_{1}, \Phi_{1}\right)\right)=\varpi\left(\left(E_{2}, \Phi_{2}\right)\right)$ if and only if $\left(E_{1}^{\vee \vee}, \Phi_{1}\right) \cong\left(E_{2}^{\vee \vee}, \Phi_{2}\right)$ and $\operatorname{Supp}\left(E_{1}^{\vee \vee} / E_{1}\right)=$ $\operatorname{Supp}\left(E_{2}^{\vee \vee} / E_{2}\right)$.

Proof. We set $E_{i}^{\prime}:=\iota\left(\left(E_{i}, \Phi_{i}\right)\right), i=1,2$. Then $\varpi\left(\left(E_{1}, \Phi_{1}\right)\right)=\varpi\left(\left(E_{2}, \Phi_{2}\right)\right)$ if and only if $\left(E_{1}^{\prime}\right)^{\vee \vee} \cong\left(E_{2}^{\prime}\right)^{\vee \vee}$ and $\operatorname{Supp}\left(\left(E_{1}^{\prime}\right)^{\vee \vee} / E_{1}^{\prime}\right)=\operatorname{Supp}\left(\left(E_{2}^{\prime}\right)^{\vee \vee} / E_{2}^{\prime}\right)$. Since $E_{i}^{\prime}, i=1,2$ fits in an exact sequence

$$
0 \rightarrow E_{i}^{\prime} \rightarrow E_{i} \rightarrow \mathcal{O}_{\ell_{\infty}}(r-1) \rightarrow 0
$$

and $E_{i}$ is locally free along $\ell_{\infty}$, we have an exact sequence

$$
0 \rightarrow\left(E_{i}^{\prime}\right)^{\vee \vee} \rightarrow E_{i}^{\vee \vee} \rightarrow \mathcal{O}_{\ell_{\infty}}(r-1) \rightarrow 0 .
$$

Moreover $E_{i}^{\vee \vee} \rightarrow \mathcal{O}_{\ell_{\infty}}(r-1)$ is uniquely determined by $\left(E_{i}^{\prime}\right)^{\vee \vee}$. Therefore our lemma holds.

We thus get the diagram (3.8) except the bottom arrow. Now the morphism $M_{0}(r, n) \rightarrow$ $M_{0}\left(r,-H, n^{\prime}\right)$ exists as $M_{0}(r, n)$ is normal by Lemma A.1. 
3.4. Proof of Proposition 3.7. In the same way, we have an immersion $\widehat{M}(r, k, \widehat{n}) \rightarrow$ $\widehat{M}\left(r, k C-H, \widehat{n}^{\prime}\right)$ and the commutative diagram (3.9).

As we already mentioned before, this implies that $-\mu(C)$ is $\widehat{\pi}^{\prime}$-nef and $\widehat{\pi}^{\prime}$-big. Here $\mu$ is defined as $\mu(\bullet):=\Delta\left(\mathcal{E}^{\prime}\right) /[\bullet]$ by the universal family $\mathcal{E}^{\prime}$ on $\widehat{\mathbb{P}}^{2} \times \widehat{M}\left(r, k C-H, \widehat{n}^{\prime}\right)$. This is enough for our purpose as two universal sheaves $\mathcal{E}$ and $\mathcal{E}^{\prime}$ are isomorphic outside $\ell_{\infty}$, so $\mu(C)$ turns out to be the same for $\mathcal{E}$ and $\mathcal{E}^{\prime}$. Thus we complete the proof of Proposition 3.7

\section{BeHAVIOR AT $\varepsilon_{1}, \varepsilon_{2}=0$}

We prove a part of Nekrasov's conjecture and derive an equation satisfied by the 'genus 0' part of the partition function in this section.

4.1. Nekrasov's conjecture. We collect some properties of $l_{\vec{\alpha}}^{\vec{k}}$.

Lemma 4.1. (1) $l_{\vec{\alpha}}^{\vec{k}}\left(\varepsilon_{1}, \varepsilon_{2}, \vec{a}\right)=l_{\vec{\alpha}}^{\vec{k}}\left(\varepsilon_{2}, \varepsilon_{1}, \vec{a}\right)$.

(2) $l_{\vec{\alpha}}^{\vec{k}}\left(\varepsilon_{1}, \varepsilon_{2}, \vec{a}\right)=\left(-e^{-\boldsymbol{\beta}\langle\vec{a}, \alpha\rangle}\right)^{\langle\vec{k}, \alpha\rangle(\langle\vec{k}, \alpha\rangle-1) / 2}\left(e^{\boldsymbol{\beta}\left(\varepsilon_{1}+\varepsilon_{2}\right)}\right)^{\langle\vec{k}, \alpha\rangle\left(\langle\vec{k}, \alpha\rangle^{2}-1\right) / 6} l_{-\vec{\alpha}}^{-\vec{k}}\left(-\varepsilon_{1},-\varepsilon_{2}, \vec{a}\right)$.

(3) $l_{\vec{\alpha}}^{\vec{k}}\left(\varepsilon_{1}, \varepsilon_{2}, \vec{a}\right)$ is regular at $\left(\varepsilon_{1}, \varepsilon_{2}\right)=0$ and

$$
l_{\vec{\alpha}}^{\vec{k}}(0,0, \vec{a})=\left(1-e^{-\boldsymbol{\beta}\langle\vec{a}, \vec{\alpha}\rangle}\right)^{\langle\vec{k}, \alpha\rangle(\langle\vec{k}, \vec{\alpha}\rangle-1) / 2} .
$$

Since

$$
\prod_{\vec{\alpha} \in \Delta}\left(-e^{-\boldsymbol{\beta}\langle\vec{a}, \vec{\alpha}\rangle}\right)^{\langle\vec{k}, \vec{\alpha}\rangle(\langle\vec{k}, \vec{\alpha}\rangle-1) / 2}=\prod_{\alpha \in \Delta_{+}} e^{\boldsymbol{\beta}\langle\vec{a}, \vec{\alpha}\rangle\langle\vec{k}, \vec{\alpha}\rangle}(-1)^{\langle\vec{k}, \vec{\alpha}\rangle}=e^{r \boldsymbol{\beta}(\vec{k}, \vec{a})}(-1)^{2\langle\vec{k}, \rho\rangle}=e^{r \boldsymbol{\beta}(\vec{k}, \vec{a})},
$$

we get

$$
\prod_{\vec{\alpha} \in \Delta} l_{\vec{\alpha}}\left(\varepsilon_{1}, \varepsilon_{2}, \vec{a}\right)=e^{r \boldsymbol{\beta}(\vec{k}, \vec{a})} \prod_{\vec{\alpha} \in \Delta} l_{-\vec{\alpha}}^{-\vec{k}}\left(-\varepsilon_{1},-\varepsilon_{2}, \vec{a}\right) .
$$

The following will be used only afterwards, but it illustrates a usage of the blowup equations in Theorem 2.4.

\section{Lemma 4.3 .}

$$
Z^{\text {inst }}\left(\varepsilon_{1},-2 \varepsilon_{1}, \vec{a} ; \mathfrak{q}, \boldsymbol{\beta}\right)=Z^{\text {inst }}\left(2 \varepsilon_{1},-\varepsilon_{1}, \vec{a} ; \mathfrak{q}, \boldsymbol{\beta}\right) .
$$

Proof. We apply the relations (2.2) and (2.5) for $d=r, 0$ after setting $\varepsilon_{2}=-\varepsilon_{1}$. We omit $\boldsymbol{\beta}$ by letting it be 1 for brevity. Then

$$
\begin{aligned}
& \sum_{\vec{k}} \frac{\mathfrak{q}^{(\vec{k}, \vec{k}) / 2}}{\prod_{\vec{\alpha} \in \Delta} l_{\vec{a}}^{\vec{k}}\left(\varepsilon_{1},-\varepsilon_{1}, \vec{a}\right)}\left(e^{(\vec{k}, \vec{a}) r} Z^{\text {inst }}\left(\varepsilon_{1},-2 \varepsilon_{1}, \vec{a}+\varepsilon_{1} \vec{k} ; t_{1}^{r / 2} \mathfrak{q}\right) Z^{\text {inst }}\left(2 \varepsilon_{1},-\varepsilon_{1}, \vec{a}-\varepsilon_{1} \vec{k} ; t_{1}^{-r / 2} \mathfrak{q}\right)\right. \\
& \left.-Z^{\text {inst }}\left(\varepsilon_{1},-2 \varepsilon_{1}, \vec{a}+\varepsilon_{1} \vec{k} ; t_{1}^{-r / 2} \mathfrak{q}\right) Z^{\text {inst }}\left(2 \varepsilon_{1},-\varepsilon_{1}, \vec{a}-\varepsilon_{1} \vec{k} ; t_{1}^{r / 2} \mathfrak{q}\right)\right)=0 .
\end{aligned}
$$

Let us expand $Z^{\text {inst }}$ as in (1.3). Then the above equation implies

$$
\begin{aligned}
&\left(Z_{n}\left(\varepsilon_{1},-2 \varepsilon_{1}, \vec{a}\right)-Z_{n}\left(2 \varepsilon_{1},-\varepsilon_{1}, \vec{a}\right)\right)\left(t_{1}^{r n / 2}-t_{1}^{-r n / 2}\right) \\
&= \sum_{\substack{(\vec{k}, \vec{k}) / 2+l+m=n \\
l \neq n, m \neq n}} \frac{e^{r(\vec{k}, \vec{a}) / 2} Z_{m}\left(\varepsilon_{1},-2 \varepsilon_{1}, \vec{a}+\varepsilon_{1} \vec{k}\right) Z_{l}\left(2 \varepsilon_{1},-\varepsilon_{1}, \vec{a}-\varepsilon_{1} \vec{k}\right)}{\prod_{\vec{\alpha} \in \Delta} l_{\vec{\alpha}}^{\vec{k}}\left(\varepsilon_{1},-\varepsilon_{1}, \vec{a}\right)} \\
& \times\left(e^{r(\vec{k}, \vec{a}) / 2} t_{1}^{r(m-l) / 2}-e^{r(-\vec{k}, \vec{a}) / 2} t_{1}^{-r(m-l) / 2}\right) .
\end{aligned}
$$

Let us show that $Z_{n}\left(\varepsilon_{1},-2 \varepsilon_{1}, \vec{a}\right)=Z_{n}\left(2 \varepsilon_{1},-\varepsilon_{1}, \vec{a}\right)$ by using the induction on $n$. It holds for $n=0$ as $Z_{0}=1$. Suppose that it is true for $l, m<n$. Then the right hand side of the above 
equation vanishes, as terms with $(\vec{k}, l, m)$ and $(-\vec{k}, m, l)$ cancel thanks to Lemma 4.1 (1) and (4.2), and the term $(0, l, l)$ is 0 . Therefore it is also true for $n$.

Now we prove a part of Nekrasov's conjecture:

Theorem 4.4. We set

$$
F^{\text {inst }}\left(\varepsilon_{1}, \varepsilon_{2}, \vec{a} ; \mathfrak{q}, \boldsymbol{\beta}\right) \stackrel{\text { def. }}{=} \varepsilon_{1} \varepsilon_{2} \log Z^{\text {inst }}\left(\varepsilon_{1}, \varepsilon_{2}, \vec{a} ; \mathfrak{q}, \boldsymbol{\beta}\right) .
$$

Then $F^{\mathrm{inst}}\left(\varepsilon_{1}, \varepsilon_{2}, \vec{a} ; \mathfrak{q}, \boldsymbol{\beta}\right)$ is regular at $\left(\varepsilon_{1}, \varepsilon_{2}\right)=(0,0)$.

Proof. We omit $\boldsymbol{\beta}$ by letting it be 1 for brevity.

By Theorem $2.4(1)$, we have $\widehat{Z}_{0, d+1}^{\text {inst }}-\widehat{Z}_{0, d}^{\text {inst }}=0$ for $0 \leq d<r$. We divide this equation by $Z^{\text {inst }}\left(\varepsilon_{1}, \varepsilon_{2}-\varepsilon_{1}, \vec{a}, t_{1}^{d-r / 2} \mathfrak{q}\right) Z^{\text {inst }}\left(\varepsilon_{1}-\varepsilon_{2}, \varepsilon_{2}, \vec{a}, t_{2}^{d-r / 2} \mathfrak{q}\right)$.

Let us expand $F^{\text {inst }}$ as

$$
F^{\text {inst }}\left(\varepsilon_{1}, \varepsilon_{2}, \vec{a} ; \mathfrak{q}\right)=\sum_{n} F_{n}\left(\varepsilon_{1}, \varepsilon_{2}, \vec{a}\right) \mathfrak{q}^{n}
$$

We note that

$$
\begin{gathered}
\frac{Z^{\text {inst }}\left(\varepsilon_{1}, \varepsilon_{2}-\varepsilon_{1}, \vec{a}+\varepsilon_{1} \vec{k} ; t_{1}^{d+1-r / 2} \mathfrak{q}\right) Z^{\text {inst }}\left(\varepsilon_{1}-\varepsilon_{2}, \varepsilon_{2}, \vec{a}+\varepsilon_{2} \vec{k} ; t_{2}^{d+1-r / 2} \mathfrak{q}\right)}{Z^{\text {inst }}\left(\varepsilon_{1}, \varepsilon_{2}-\varepsilon_{1}, \vec{a} ; t_{1}^{d-r / 2} \mathfrak{q}\right) Z^{\text {inst }}\left(\varepsilon_{1}-\varepsilon_{2}, \varepsilon_{2}, \vec{a} ; t_{2}^{d-r / 2} \mathfrak{q}\right)} \\
=\frac{Z^{\text {inst }}\left(\varepsilon_{1}, \varepsilon_{2}-\varepsilon_{1}, \vec{a}+\varepsilon_{1} \vec{k} ; t_{1}^{d+1-r / 2} \mathfrak{q}\right) Z^{\text {inst }}\left(\varepsilon_{1}-\varepsilon_{2}, \varepsilon_{2}, \vec{a}+\varepsilon_{2} \vec{k} ; t_{2}^{d+1-r / 2} \mathfrak{q}\right)}{Z^{\text {inst }}\left(\varepsilon_{1}, \varepsilon_{2}-\varepsilon_{1}, \vec{a} ; t_{1}^{d+1-r / 2} \mathfrak{q}\right) Z^{\text {inst }}\left(\varepsilon_{1}-\varepsilon_{2}, \varepsilon_{2}, \vec{a} ; t_{2}^{d+1-r / 2} \mathfrak{q}\right)} \\
\quad \times \frac{Z^{\text {inst }}\left(\varepsilon_{1}, \varepsilon_{2}-\varepsilon_{1}, \vec{a} ; t_{1}^{d+1-r / 2} \mathfrak{q}\right) Z^{\text {inst }}\left(\varepsilon_{1}-\varepsilon_{2}, \varepsilon_{2}, \vec{a} ; t_{2}^{d+1-r / 2} \mathfrak{q}\right)}{Z^{\text {inst }}\left(\varepsilon_{1}, \varepsilon_{2}-\varepsilon_{1}, \vec{a} ; t_{1}^{d-r / 2} \mathfrak{q}\right) Z^{\text {inst }}\left(\varepsilon_{1}-\varepsilon_{2}, \varepsilon_{2}, \vec{a} ; t_{2}^{d-r / 2} \mathfrak{q}\right)} \\
=\exp \left[\sum _ { n \geq 1 } \left(\frac{F_{n}^{\text {inst }}\left(\varepsilon_{1}, \varepsilon_{2}-\varepsilon_{1}, \vec{a}+\varepsilon_{1} \vec{k}\right)-F_{n}^{\text {inst }}\left(\varepsilon_{1}, \varepsilon_{2}-\varepsilon_{1}, \vec{a}\right)}{\left.\varepsilon_{1}-\varepsilon_{1}\right)} t_{1}^{(d+1-r / 2) n}\right.\right. \\
\left.+\frac{F_{n}^{\text {inst }}\left(\varepsilon_{1}-\varepsilon_{2}, \varepsilon_{2}, \vec{a}+\varepsilon_{1} \vec{k}\right)-F_{n}^{\text {inst }}\left(\varepsilon_{1}-\varepsilon_{2}, \varepsilon_{2}, \vec{a}\right)}{\varepsilon_{2}\left(\varepsilon_{2}-\varepsilon_{1}\right)} t_{2}^{(d+1-r / 2) n}\right) \mathfrak{q}^{n} \\
\left.+\sum_{n \geq 1}\left(\frac{F_{n}^{\text {inst }}\left(\varepsilon_{1}, \varepsilon_{2}-\varepsilon_{1}, \vec{a}\right)\left(t_{1}^{n}-1\right) t_{1}^{(d-r / 2) n}}{\varepsilon_{1}\left(\varepsilon_{2}-\varepsilon_{1}\right)}-\frac{F_{n}^{\text {inst }}\left(\varepsilon_{1}-\varepsilon_{2}, \varepsilon_{2}, \vec{a}\right)\left(t_{2}^{n}-1\right) t_{2}^{(d-r / 2) n}}{\varepsilon_{2}\left(\varepsilon_{2}-\varepsilon_{1}\right)}\right) \mathfrak{q}^{n}\right] .
\end{gathered}
$$

By (2.2) we get the following relations for $d=0,1$ :

$$
\begin{gathered}
\sum_{n \geq 1}\left(\frac{F_{n}^{\text {inst }}\left(\varepsilon_{1}, \varepsilon_{2}-\varepsilon_{1}, \vec{a}\right)\left(t_{1}^{n}-1\right) t_{1}^{-r n / 2}}{\varepsilon_{1}\left(\varepsilon_{2}-\varepsilon_{1}\right)}-\frac{F_{n}^{\text {inst }}\left(\varepsilon_{1}-\varepsilon_{2}, \varepsilon_{2}, \vec{a}\right)\left(t_{2}^{n}-1\right) t_{2}^{-r n / 2}}{\varepsilon_{2}\left(\varepsilon_{2}-\varepsilon_{1}\right)}\right) \mathfrak{q}^{n}=\sum_{n} A_{n} \mathfrak{q}^{n} \\
\sum_{n \geq 1}\left(\frac{F_{n}^{\text {inst }}\left(\varepsilon_{1}, \varepsilon_{2}-\varepsilon_{1}, \vec{a}\right)\left(t_{1}^{n}-1\right) t_{1}^{(1-r / 2) n}}{\varepsilon_{1}\left(\varepsilon_{2}-\varepsilon_{1}\right)}-\frac{F_{n}^{\text {inst }}\left(\varepsilon_{1}-\varepsilon_{2}, \varepsilon_{2}, \vec{a}\right)\left(t_{2}^{n}-1\right) t_{2}^{(1-r / 2) n}}{\varepsilon_{2}\left(\varepsilon_{2}-\varepsilon_{1}\right)}\right) \mathfrak{q}^{n}=\sum_{n} B_{n} \mathfrak{q}^{n}
\end{gathered}
$$

where the right hand sides comes from terms with $\vec{k} \neq 0$. In particular, $A_{n}$ and $B_{n}$ are written by $F_{m}$ with $m<n$. Solving the above, we get

$$
F_{n}\left(\varepsilon_{1}, \varepsilon_{2}-\varepsilon_{1}, \vec{a}\right)=\frac{\varepsilon_{1}\left(\varepsilon_{2}-\varepsilon_{1}\right)}{\left(t_{1}^{n}-1\right)\left(t_{2}^{n}-t_{1}^{n}\right)} t_{1}^{r n / 2}\left(t_{2}^{n} A_{n}-B_{n}\right) .
$$


Hence if $F_{m}, m<n$ are regular at $\left(\varepsilon_{1}, \varepsilon_{2}\right)=(0,0)$, then $F_{n}$ is also regular. As $F_{0}=0$, we get the assertion by the induction on $n$.

4.2. The perturbative part. We set

$$
\begin{aligned}
& \quad \gamma_{\varepsilon_{1}, \varepsilon_{2}}(x \mid \boldsymbol{\beta} ; \Lambda) \stackrel{\text { def. }}{=} \frac{1}{2 \varepsilon_{1} \varepsilon_{2}}\left(-\frac{\boldsymbol{\beta}}{6}\left(x+\frac{1}{2}\left(\varepsilon_{1}+\varepsilon_{2}\right)\right)^{3}+x^{2} \log (\boldsymbol{\beta} \Lambda)\right)+\sum_{n \geq 1} \frac{1}{n} \frac{e^{-\boldsymbol{\beta} n x}}{\left(e^{\boldsymbol{\beta} n \varepsilon_{1}}-1\right)\left(e^{\boldsymbol{\beta} n \varepsilon_{2}}-1\right)}, \\
& \quad \widetilde{\gamma}_{\varepsilon_{1}, \varepsilon_{2}}(x \mid \boldsymbol{\beta} ; \Lambda) \\
& \stackrel{\text { def. }}{=} \gamma_{\varepsilon_{1}, \varepsilon_{2}}(x \mid \boldsymbol{\beta} ; \Lambda)+\frac{1}{\varepsilon_{1} \varepsilon_{2}}\left(\frac{\pi^{2} x}{6 \boldsymbol{\beta}}-\frac{\zeta(3)}{\boldsymbol{\beta}^{2}}\right)+\frac{\varepsilon_{1}+\varepsilon_{2}}{2 \varepsilon_{1} \varepsilon_{2}}\left(x \log (\boldsymbol{\beta} \Lambda)+\frac{\pi^{2}}{6 \boldsymbol{\beta}}\right)+\frac{\varepsilon_{1}^{2}+\varepsilon_{2}^{2}+3 \varepsilon_{1} \varepsilon_{2}}{12 \varepsilon_{1} \varepsilon_{2}} \log (\boldsymbol{\beta} \Lambda)
\end{aligned}
$$

for $\boldsymbol{\beta} x>0$. Here $\Lambda=\mathfrak{q}^{1 / 2 r}$. If we formally expand $\varepsilon_{1} \varepsilon_{2} \widetilde{\gamma}_{\varepsilon_{1}, \varepsilon_{2}}(x \mid \boldsymbol{\beta} ; \Lambda)$ as a power series of $\varepsilon_{1}, \varepsilon_{2}$ around $\varepsilon_{1}=\varepsilon_{2}=0$, then each coefficient is a holomorphic function of $x$. Indeed if we expand

$$
\frac{1}{\left(e^{\varepsilon_{1} \boldsymbol{\beta}}-1\right)\left(e^{\varepsilon_{2} \boldsymbol{\beta}}-1\right)}=\sum_{m \geq 0} \frac{c_{m}}{m !} \boldsymbol{\beta}^{m-2},
$$

then

$$
\sum_{n \geq 1} \frac{1}{n} \frac{e^{-\boldsymbol{\beta} n x}}{\left(e^{\boldsymbol{\beta} n \varepsilon_{1}}-1\right)\left(e^{\boldsymbol{\beta} n \varepsilon_{2}}-1\right)}=\sum_{m \geq 0} \frac{c_{m}}{m !} \boldsymbol{\beta}^{m-2} \operatorname{Li}_{3-m}\left(e^{-\boldsymbol{\beta} x}\right),
$$

where $\mathrm{Li}_{3-m}\left(e^{-\boldsymbol{\beta} x}\right)$ are polylogarithms.

We extend the definition of $\gamma_{\varepsilon_{1}, \varepsilon_{2}}(x \mid \boldsymbol{\beta} ; \Lambda)$ to the range $\boldsymbol{\beta} x<0$ by analytic continuation along a circle counter-clockwise way.

Proposition 4.5. If $\vec{a}$ is in a neighborhood of the region $\boldsymbol{\beta}\langle\vec{a}, \vec{\alpha}\rangle>0$ for all $\vec{\alpha} \in \Delta_{+}$,

$$
\begin{gathered}
\sum_{\vec{\alpha} \in \Delta}\left(\widetilde{\gamma}_{\varepsilon_{1}, \varepsilon_{2}-\varepsilon_{1}}\left(\left\langle\vec{a}+\vec{k} \varepsilon_{1}, \vec{\alpha}\right\rangle \mid \boldsymbol{\beta} ; \Lambda e^{(d-r / 2) \boldsymbol{\beta} \varepsilon_{1} / 2 r}\right)\right. \\
\left.+\widetilde{\gamma}_{\varepsilon_{1}-\varepsilon_{2}, \varepsilon_{2}}\left(\left\langle\vec{a}+\vec{k} \varepsilon_{2}, \vec{\alpha}\right\rangle \mid \boldsymbol{\beta} ; \Lambda e^{(d-r / 2) \boldsymbol{\beta} \varepsilon_{2} / 2 r}\right)-\widetilde{\gamma}_{\varepsilon_{1}, \varepsilon_{2}}(\langle\vec{a}, \vec{\alpha}\rangle \mid \boldsymbol{\beta} ; \Lambda)\right) \\
=-\frac{(4 d-r)(r-1)}{48}\left(\varepsilon_{1}+\varepsilon_{2}\right) \boldsymbol{\beta}-\frac{(\vec{k}, \vec{k})}{2}\left(\log \left((\boldsymbol{\beta} \Lambda)^{2 r}\right)-\left(d-\frac{r}{2}\right)\left(\varepsilon_{1}+\varepsilon_{2}\right) \boldsymbol{\beta}\right) \\
-d(\vec{k}, \vec{a}) \boldsymbol{\beta}+\sum_{\vec{\alpha} \in \Delta} \log \left(l_{\vec{\alpha}}^{\vec{k}}\left(\varepsilon_{1}, \varepsilon_{2}, \vec{a}\right)\right) .
\end{gathered}
$$

Proof. If $k=-l$ with $l>0$, then

$$
\begin{aligned}
& \frac{e^{-\left(x+k \varepsilon_{1}\right)}}{\left(e^{\varepsilon_{1}}-1\right)\left(e^{\varepsilon_{2}-\varepsilon_{1}}-1\right)}+\frac{e^{-\left(x+k \varepsilon_{2}\right)}}{\left(e^{\varepsilon_{2}}-1\right)\left(e^{\varepsilon_{1}-\varepsilon_{2}}-1\right)}-\frac{e^{-x}}{\left(e^{\varepsilon_{1}}-1\right)\left(e^{\varepsilon_{2}}-1\right)} \\
= & e^{-x}\left(\frac{e^{\varepsilon_{1}+\varepsilon_{2}}\left(e^{l \varepsilon_{1}}-e^{l \varepsilon_{2}}\right)-\left(e^{(l+1) \varepsilon_{1}}-e^{(l+1) \varepsilon_{2}}\right)}{\left(e^{\varepsilon_{1}}-1\right)\left(e^{\varepsilon_{2}}-1\right)\left(e^{\varepsilon_{2}}-e^{\varepsilon_{1}}\right)}-\frac{1}{\left(e^{\varepsilon_{1}}-1\right)\left(e^{\varepsilon_{2}}-1\right)}\right) \\
= & e^{-x}\left(\frac{-\sum_{i=0}^{l-1} e^{(i+1) \varepsilon_{1}} e^{(l-i) \varepsilon_{2}}+\sum_{i=0}^{l} e^{i \varepsilon_{1}} e^{(l-i) \varepsilon_{2}}}{\left(e^{\varepsilon_{1}}-1\right)\left(e^{\varepsilon_{2}}-1\right)}-\frac{1}{\left(e^{\varepsilon_{1}}-1\right)\left(e^{\varepsilon_{2}}-1\right)}\right) \\
= & e^{-x} \frac{-\sum_{i=0}^{l-1} e^{i \varepsilon_{1}} e^{(l-i) \varepsilon_{2}}+\sum_{i=0}^{l-1} e^{i \varepsilon_{1}}}{\left(e^{\varepsilon_{2}}-1\right)}=-e^{-x} \sum_{\substack{0 \leq i, j \\
i+j \leq l-1}} e^{i \varepsilon_{1}} e^{j \varepsilon_{2}} .
\end{aligned}
$$


Since

$$
\frac{\varepsilon_{1} \varepsilon_{2} e^{-t x}}{\left(e^{t \varepsilon_{1}}-1\right)\left(e^{t \varepsilon_{2}}-1\right)}
$$

is holomorphic at $\left(\varepsilon_{1}, \varepsilon_{2}\right)=(0,0)$, (4.6) holds in $\mathbb{C}\left[e^{-x}\right]\left[\left[\varepsilon_{1}, \varepsilon_{2}\right]\right]\left[\frac{1}{\varepsilon_{1} \varepsilon_{2}\left(\varepsilon_{1}-\varepsilon_{2}\right)}\right]$.

If $k>0$, then

$$
\begin{aligned}
& \frac{e^{-\left(x+k \varepsilon_{1}\right)}}{\left(e^{\varepsilon_{1}}-1\right)\left(e^{\varepsilon_{2}-\varepsilon_{1}}-1\right)}+\frac{e^{-\left(x+k \varepsilon_{2}\right)}}{\left(e^{\varepsilon_{2}}-1\right)\left(e^{\varepsilon_{1}-\varepsilon_{2}}-1\right)}-\frac{e^{-x}}{\left(e^{\varepsilon_{1}}-1\right)\left(e^{\varepsilon_{2}}-1\right)} \\
= & e^{-x-\varepsilon_{1}-\varepsilon_{2}}\left(\frac{\left(e^{-k \varepsilon_{1}}-e^{-k \varepsilon_{2}}\right)-\left(e^{-(k-1) \varepsilon_{1}}-e^{-(k-1) \varepsilon_{2}}\right) e^{-\varepsilon_{1}-\varepsilon_{2}}}{\left(e^{-\varepsilon_{1}}-1\right)\left(e^{-\varepsilon_{2}}-1\right)\left(e^{-\varepsilon_{1}}-e^{-\varepsilon_{2}}\right)}-\frac{1}{\left(e^{-\varepsilon_{1}}-1\right)\left(e^{-\varepsilon_{2}}-1\right)}\right) \\
= & e^{-x}\left(\frac{\sum_{i=0}^{k-1} e^{-i \varepsilon_{1}} e^{-(k-1-i) \varepsilon_{2}}-\sum_{i=0}^{k-2} e^{-(i+1) \varepsilon_{1}} e^{-(k-1-i) \varepsilon_{2}}}{\left(e^{-\varepsilon_{1}}-1\right)\left(e^{-\varepsilon_{2}}-1\right)}-\frac{1}{\left(e^{-\varepsilon_{1}}-1\right)\left(e^{-\varepsilon_{2}}-1\right)}\right) \\
= & e^{-x \frac{-\sum_{i=0}^{k-2} e^{i \varepsilon_{1}} e^{-(k-1-i) \varepsilon_{2}}+\sum_{i=0}^{k-2} e^{-i \varepsilon_{1}}}{\left(e^{-\varepsilon_{2}}-1\right)}=-e^{-x} \sum_{\substack{0 \leq i, j \\
i+j \leq k-2}} e^{-(i+1) \varepsilon_{1}} e^{-(j+1) \varepsilon_{2}} .}
\end{aligned}
$$

Hence

$$
\begin{aligned}
& \left.\sum_{n \geq 1} \frac{1}{n} \frac{e^{-\boldsymbol{\beta} n(\vec{a}, \vec{\alpha})}}{\left(e^{\boldsymbol{\beta} n \varepsilon_{1}}-1\right)\left(e^{\boldsymbol{\beta} n \varepsilon_{2}}-1\right)}\right|_{\substack{\vec{a} \rightarrow \vec{a}+\vec{k} \varepsilon_{1} \\
\varepsilon_{1} \rightarrow \varepsilon_{1}}}+\left.\sum_{n \geq 1} \frac{1}{n} \frac{e^{-\boldsymbol{\beta} n(\vec{a}, \vec{\alpha})}}{\left(e^{\boldsymbol{\beta} n \varepsilon_{1}}-1\right)\left(e^{t n \varepsilon_{2}}-1\right)}\right|_{\substack{\vec{a} \rightarrow \vec{a}+\vec{k} \varepsilon_{2} \\
\varepsilon_{1} \rightarrow \varepsilon_{1}-\varepsilon_{2} \\
\varepsilon_{2} \rightarrow \varepsilon_{2}}} \\
& -\sum_{n \geq 1} \frac{1}{n} \frac{e^{-\boldsymbol{\beta} n(\vec{a}, \vec{\alpha})}}{\left(e^{\boldsymbol{\beta} n \varepsilon_{1}}-1\right)\left(e^{\boldsymbol{\beta} n \varepsilon_{2}}-1\right)}=\log \left(l_{\vec{\alpha}}^{\vec{k}}\left(\varepsilon_{1}, \varepsilon_{2}, \vec{a}\right)\right)
\end{aligned}
$$

in $\mathcal{O}\left[\left[\varepsilon_{1}, \varepsilon_{2}\right]\right]\left[\frac{1}{\varepsilon_{1} \varepsilon_{2}\left(\varepsilon_{1}-\varepsilon_{2}\right)}\right]$, where $\mathcal{O}$ is the ring of holomorphic functions of $\boldsymbol{\beta} \vec{a}$ in a neighborhood of $\boldsymbol{\beta}\langle\vec{a}, \vec{\alpha}\rangle>0$. By the analytic continuation, (4.7) also holds for the range $\boldsymbol{\beta}\langle\vec{a}, \vec{\alpha}\rangle<0$. Combining the following equalities, we get our claim.

$$
\begin{aligned}
& \frac{-\left(x+\varepsilon_{1} k+\frac{1}{2} \varepsilon_{2}\right)^{3}}{12 \varepsilon_{1}\left(\varepsilon_{2}-\varepsilon_{1}\right)}+\frac{-\left(x+\varepsilon_{2} k+\frac{1}{2} \varepsilon_{1}\right)^{3}}{12 \varepsilon_{2}\left(\varepsilon_{1}-\varepsilon_{2}\right)} \\
&= \frac{-\left(x+\frac{1}{2}\left(\varepsilon_{1}+\varepsilon_{2}\right)\right)^{3}}{12 \varepsilon_{1} \varepsilon_{2}}+\frac{4 k^{2}-4 k+1}{16} x+\frac{8 k^{3}-6 k+2}{96}\left(\varepsilon_{1}+\varepsilon_{2}\right), \\
& \frac{\left(x+k \varepsilon_{1}\right)^{2}}{2 \varepsilon_{1}\left(\varepsilon_{2}-\varepsilon_{1}\right)} \log \left(\boldsymbol{\beta} \Lambda e^{(d-r / 2) \boldsymbol{\beta} \varepsilon_{1} / 2 r}\right)+\frac{\left(x+k \varepsilon_{2}\right)^{2}}{2 \varepsilon_{2}\left(\varepsilon_{1}-\varepsilon_{2}\right)} \log \left(\boldsymbol{\beta} \Lambda e^{(d-r / 2) \boldsymbol{\beta} \varepsilon_{2} / 2 r}\right) \\
&= \frac{x^{2}}{2 \varepsilon_{1} \varepsilon_{2}} \log (\boldsymbol{\beta} \Lambda)-\frac{k^{2}}{2} \log (\boldsymbol{\beta} \Lambda)-\frac{d-\frac{r}{2}}{2 r}\left(\frac{k^{2}}{2}\left(\varepsilon_{1}+\varepsilon_{2}\right)+k x\right), \\
& \frac{\pi^{2}\left(x+k \varepsilon_{1}\right)}{6 \boldsymbol{\beta} \varepsilon_{1}\left(\varepsilon_{2}-\varepsilon_{1}\right)}+\frac{\pi^{2}\left(x+k \varepsilon_{2}\right)}{6 \boldsymbol{\beta}\left(\varepsilon_{1}-\varepsilon_{2}\right) \varepsilon_{2}}=\frac{\pi^{2} x}{6 \boldsymbol{\beta} \varepsilon_{1} \varepsilon_{2}}, \\
& \quad \frac{\varepsilon_{2}\left(x+k \varepsilon_{1}\right) \log \left(\boldsymbol{\beta} \Lambda e^{(d-r / 2) \boldsymbol{\beta} \varepsilon_{1} / 2 r}\right)}{2 \varepsilon_{1}\left(\varepsilon_{2}-\varepsilon_{1}\right)}+\frac{\varepsilon_{1}\left(x+k \varepsilon_{2}\right)}{\log \left(\boldsymbol{\beta} \Lambda e^{(d-r / 2) \boldsymbol{\beta} \varepsilon_{2} / 2 r}\right)} \\
&= \frac{\left(\varepsilon_{1}+\varepsilon_{2}\right) x \log (\boldsymbol{\beta} \Lambda)}{2 \varepsilon_{1} \varepsilon_{2}}+\frac{k}{2} \log (\boldsymbol{\beta} \Lambda)+\frac{\left(d-\frac{r}{2}\right) \boldsymbol{\beta} x}{4 r}
\end{aligned}
$$




$$
\begin{aligned}
& \frac{-\varepsilon_{1}^{2}+\varepsilon_{2}^{2}+\varepsilon_{1} \varepsilon_{2}}{12 \varepsilon_{1}\left(\varepsilon_{2}-\varepsilon_{1}\right)} \log \left(\boldsymbol{\beta} \Lambda e^{(d-r / 2) \boldsymbol{\beta} \varepsilon_{1} / 2 r}\right)+\frac{\varepsilon_{1}^{2}-\varepsilon_{2}^{2}+\varepsilon_{1} \varepsilon_{2}}{12\left(\varepsilon_{1}-\varepsilon_{2}\right) \varepsilon_{2}} \log \left(\boldsymbol{\beta} \Lambda e^{(d-r / 2) \boldsymbol{\beta} \varepsilon_{2} / 2 r}\right) \\
= & \frac{\varepsilon_{1}^{2}+\varepsilon_{2}^{2}+3 \varepsilon_{1} \varepsilon_{2}}{\varepsilon_{1} \varepsilon_{2}} \log (\boldsymbol{\beta} \Lambda)+\frac{\left(d-\frac{r}{2}\right) \boldsymbol{\beta}\left(\varepsilon_{1}+\varepsilon_{2}\right)}{12 r} .
\end{aligned}
$$

By using (B.6) and (B.8), we see that

$$
\begin{aligned}
& \varepsilon_{1} \varepsilon_{2}(\widetilde{\gamma}(x ; \boldsymbol{\beta})+\widetilde{\gamma}(-x ; \boldsymbol{\beta})) \\
=2 & \left(\frac{1}{\boldsymbol{\beta}^{2}}\left(\operatorname{Li}_{3}\left(e^{-\boldsymbol{\beta} x}\right)-\zeta(3)\right)+\frac{x^{2}}{2} \log (\boldsymbol{\beta} \Lambda)+\frac{\pi^{2} x}{6 \boldsymbol{\beta}}\right)-\frac{x^{2} \pi \sqrt{-1}}{2}-\frac{\boldsymbol{\beta} x^{3}}{6} \\
& +\left(\varepsilon_{1}+\varepsilon_{2}\right) \frac{x}{2} \pi \sqrt{-1}-\frac{\varepsilon_{1}^{2}+\varepsilon_{2}^{2}+3 \varepsilon_{1} \varepsilon_{2}}{6}\left(\log \left(\frac{1-e^{-\boldsymbol{\beta} x}}{\boldsymbol{\beta} \Lambda}\right)+\frac{\boldsymbol{\beta} x+\pi \sqrt{-1}}{2}\right)+\cdots .
\end{aligned}
$$

By $\$$ B.3. we get

$$
\begin{aligned}
& \varepsilon_{1} \varepsilon_{2}(\widetilde{\gamma}(x ; \boldsymbol{\beta})+\widetilde{\gamma}(-x ; \boldsymbol{\beta})) \\
& \underset{\boldsymbol{\beta} \rightarrow 0}{\longrightarrow}\left(-\frac{1}{2} x^{2} \log \left(\frac{x}{\Lambda}\right)+\frac{3}{4} x^{2}\right)+\left(-\frac{1}{2} x^{2} \log \left(\frac{-x}{\Lambda}\right)+\frac{3}{4} x^{2}\right) \\
& \quad+\left(\varepsilon_{1}+\varepsilon_{2}\right) \frac{x}{2} \pi \sqrt{-1}-\frac{\varepsilon_{1}^{2}+\varepsilon_{2}^{2}+3 \varepsilon_{1} \varepsilon_{2}}{6}\left(\log \left(\frac{x}{\Lambda}\right)+\frac{\pi \sqrt{-1}}{2}\right)+\cdots
\end{aligned}
$$

Therefore the perturbative part of the $K$-theoretic partition function (see below) converges to that of the homological partition function as $\boldsymbol{\beta} \rightarrow 0$.

4.3. The full partition functions. By adding the perturbative term, we define the full partition functions as

$$
\begin{aligned}
& Z\left(\varepsilon_{1}, \varepsilon_{2}, \vec{a} ; \mathfrak{q}, \boldsymbol{\beta}\right) \stackrel{\text { def. }}{=} \exp \left(\sum_{\vec{\alpha} \in \Delta}-\widetilde{\gamma}_{\varepsilon_{1}, \varepsilon_{2}}(\langle\vec{a}, \vec{\alpha}\rangle \mid \boldsymbol{\beta} ; \Lambda)\right) Z^{\text {inst }}\left(\varepsilon_{1}, \varepsilon_{2}, \vec{a} ; \mathfrak{q}, \boldsymbol{\beta}\right) \\
& \widehat{Z}_{k, d}\left(\varepsilon_{1}, \varepsilon_{2}, \vec{a} ; \mathfrak{q}, \boldsymbol{\beta}\right) \stackrel{\text { def. }}{=} \exp \left(\sum_{\vec{\alpha} \in \Delta}-\widetilde{\gamma}_{\varepsilon_{1}, \varepsilon_{2}}(\langle\vec{a}, \vec{\alpha}\rangle \mid \boldsymbol{\beta} ; \Lambda)\right) Z_{k, d}^{\text {inst }}\left(\varepsilon_{1}, \varepsilon_{2}, \vec{a} ; \mathfrak{q}, \boldsymbol{\beta}\right) .
\end{aligned}
$$

By (2.2) and Proposition 4.5, we get

$$
\begin{aligned}
& \widehat{Z}_{k, d}\left(\varepsilon_{1}, \varepsilon_{2}, \vec{a} ; \mathfrak{q}, \boldsymbol{\beta}\right)=\sum_{\{\vec{k}\}=-k / d} \exp \left[-\frac{(4 d-r)(r-1)}{48} \boldsymbol{\beta}\left(\varepsilon_{1}+\varepsilon_{2}\right)\right] \\
& \times Z\left(\varepsilon_{1}, \varepsilon_{2}-\varepsilon_{1}, \vec{a}+\varepsilon_{1} \vec{k} ; t_{1}^{\left(d-\frac{r}{2}\right)} \mathfrak{q}, \boldsymbol{\beta}\right) Z\left(\varepsilon_{1}-\varepsilon_{2}, \varepsilon_{2}, \vec{a}+\varepsilon_{2} \vec{k} ; t_{2}^{\left(d-\frac{r}{2}\right)} \mathfrak{q}, \boldsymbol{\beta}\right) .
\end{aligned}
$$

If we set $\varepsilon_{2}=-\varepsilon_{1}$, then

$$
\widehat{Z}_{k, d}\left(\varepsilon_{1},-\varepsilon_{1}, \vec{a} ; \mathfrak{q}, \boldsymbol{\beta}\right)=\sum_{\{\vec{k}\}=-k / d} Z\left(\varepsilon_{1},-2 \varepsilon_{1}, \vec{a}+\varepsilon_{1} \vec{k} ; t_{1}^{\left(d-\frac{r}{2}\right)} \mathfrak{q}, \boldsymbol{\beta}\right) Z\left(2 \varepsilon_{1},-\varepsilon_{1}, \vec{a}-\varepsilon_{1} \vec{k} ; t_{1}^{-\left(d-\frac{r}{2}\right)} \mathfrak{q}, \boldsymbol{\beta}\right) .
$$

We expand $F$ as

$$
\begin{aligned}
& F\left(\varepsilon_{1}, \varepsilon_{2}, \vec{a} ; \mathfrak{q}, \boldsymbol{\beta}\right)=\varepsilon_{1} \varepsilon_{2} \log Z\left(\varepsilon_{1}, \varepsilon_{2}, \vec{a} ; \mathfrak{q}, \boldsymbol{\beta}\right) \\
= & F_{0}(\vec{a} ; \mathfrak{q}, \boldsymbol{\beta})+\left(\varepsilon_{1}+\varepsilon_{2}\right) H(\vec{a} ; \mathfrak{q}, \boldsymbol{\beta})+\left(\varepsilon_{1}+\varepsilon_{2}\right)^{2} G(\vec{a} ; \mathfrak{q}, \boldsymbol{\beta})+\varepsilon_{1} \varepsilon_{2} F_{1}(\vec{a} ; \mathfrak{q}, \boldsymbol{\beta})+\cdots .
\end{aligned}
$$


By Lemma 4.3, $H(\vec{a} ; \mathfrak{q}, \boldsymbol{\beta})$ comes from the perturbative part:

$$
\begin{aligned}
& H(\vec{a} ; \mathfrak{q}, \boldsymbol{\beta})=\sum_{\alpha \neq \beta}\left(\frac{1}{2 \boldsymbol{\beta}} \operatorname{Li}_{2}\left(e^{-\boldsymbol{\beta}\left(a_{\alpha}-a_{\beta}\right)}\right)+\frac{\boldsymbol{\beta}}{8}\left(a_{\alpha}-a_{\beta}\right)^{2}-\frac{1}{2}\left(a_{\alpha}-a_{\beta}\right) \log (\boldsymbol{\beta} \Lambda)-\frac{\pi^{2}}{12 \boldsymbol{\beta}}\right) \\
= & -\sum_{\alpha<\beta} \pi \sqrt{-1} \frac{\left(a_{\alpha}-a_{\beta}\right)}{2}=-\pi \sqrt{-1}\langle\vec{a}, \rho\rangle .
\end{aligned}
$$

Let us derive equations for $F_{0}$. We use

$$
\begin{aligned}
& \frac{F_{0}\left(\vec{a}+\varepsilon_{1} \vec{k} ; t_{1}^{(d-r / 2)} \mathfrak{q}, \boldsymbol{\beta}\right)}{\varepsilon_{1}\left(\varepsilon_{2}-\varepsilon_{1}\right)}+\frac{F_{0}\left(\vec{a}+\varepsilon_{2} \vec{k} ; t_{2}^{(d-r / 2)} \mathfrak{q}, \boldsymbol{\beta}\right)}{\left(\varepsilon_{1}-\varepsilon_{2}\right) \varepsilon_{2}} \\
& =\frac{1}{\varepsilon_{1} \varepsilon_{2}} F_{0}-\left[\frac{\partial^{2} F_{0}}{(\partial \log \mathfrak{q})^{2}} \frac{\boldsymbol{\beta}^{2}}{2}\left(d-\frac{r}{2}\right)^{2}+\frac{\partial^{2} F_{0}}{\partial \log \mathfrak{q} \partial a^{l}} \boldsymbol{\beta}\left(d-\frac{r}{2}\right) k^{l}+\frac{\partial^{2} F_{0}}{\partial a^{l} \partial a^{m}} \frac{k^{l} k^{m}}{2}\right]+\cdots, \\
& \frac{\varepsilon_{2} H\left(\vec{a}+\varepsilon_{1} \vec{k} ; t_{1}^{(d-r / 2)} \mathfrak{q}, \boldsymbol{\beta}\right)}{\varepsilon_{1}\left(\varepsilon_{2}-\varepsilon_{1}\right)}+\frac{\varepsilon_{1} H\left(\vec{a}+\varepsilon_{2} \vec{k} ; t_{2}^{(d-r / 2)} \mathfrak{q}, \boldsymbol{\beta}\right)}{\left(\varepsilon_{1}-\varepsilon_{2}\right) \varepsilon_{2}} \\
& =\frac{\varepsilon_{1}+\varepsilon_{2}}{\varepsilon_{1} \varepsilon_{2}} H+\left[\frac{\partial H}{\partial \log \mathfrak{q}} \boldsymbol{\beta}\left(d-\frac{r}{2}\right)+\frac{\partial H}{\partial a^{l}} k^{l}\right]+\cdots=\frac{\varepsilon_{1}+\varepsilon_{2}}{\varepsilon_{1} \varepsilon_{2}} H-\pi \sqrt{-1}\langle\vec{k}, \rho\rangle+\cdots, \\
& \frac{\varepsilon_{2}^{2} G\left(\vec{a}+\varepsilon_{1} \vec{k} ; t_{1}^{\left(d-\frac{1}{r}\right)} \mathfrak{q}, \boldsymbol{\beta}\right)}{\varepsilon_{1}\left(\varepsilon_{2}-\varepsilon_{1}\right)}+\frac{\varepsilon_{1}^{2} G\left(\vec{a}+\varepsilon_{2} \vec{k} ; t_{2}^{\left(d-\frac{1}{r}\right)} \mathfrak{q}, \boldsymbol{\beta}\right)}{\left(\varepsilon_{1}-\varepsilon_{2}\right) \varepsilon_{2}}=\frac{\left(\varepsilon_{1}+\varepsilon_{2}\right)^{2}-\varepsilon_{1} \varepsilon_{2}}{\varepsilon_{1} \varepsilon_{2}} G+\cdots, \\
& F_{1}\left(\vec{a}+\varepsilon_{1} \vec{k} ; t_{1}^{\left(d-\frac{1}{r}\right)} \mathfrak{q}, \boldsymbol{\beta}\right)+F_{1}\left(\vec{a}+\varepsilon_{2} \vec{k} ; t_{2}^{\left(d-\frac{1}{r}\right)} \mathfrak{q}, \boldsymbol{\beta}\right)=2 F_{1}+\cdots,
\end{aligned}
$$

where $F_{0}, H, G, F_{1}$ and their derivatives are evaluated at $\vec{a}, \mathfrak{q}$ in the right hand sides.

By Theorem 2.4(1) and (4.14), we have

$$
\begin{aligned}
& \exp \left(F_{1}\right)=\exp \left(2 F_{1}-G\right) \sum_{\{\vec{k}\}=0}(-1)^{-\langle\vec{k}, \rho\rangle} \times \\
& \exp \left[-\frac{\partial^{2} F_{0}}{(\partial \log \mathfrak{q})^{2}} \frac{\boldsymbol{\beta}^{2}}{2}\left(d-\frac{r}{2}\right)^{2}-\frac{\partial^{2} F_{0}}{\partial \log \mathfrak{q} \partial a^{l}} \boldsymbol{\beta}\left(d-\frac{r}{2}\right) k^{l}-\frac{\partial^{2} F_{0}}{\partial a^{l} \partial a^{m}} \frac{k^{l} k^{m}}{2}\right]
\end{aligned}
$$

for $0 \leq d \leq r$

We set

$$
\tau_{l m}(\boldsymbol{\beta}) \stackrel{\text { def. }}{=}-\frac{1}{2 \pi \sqrt{-1}} \frac{\partial^{2} F_{0}(\vec{a} ; \mathfrak{q}, \boldsymbol{\beta})}{\partial a^{l} \partial a^{m}} .
$$

Then (4.15) can be written as

$$
\exp \left(G-F_{1}\right)=\exp \left[-\frac{\partial^{2} F_{0}}{(\partial \log \mathfrak{q})^{2}} \frac{\boldsymbol{\beta}^{2}}{2}\left(d-\frac{r}{2}\right)^{2}\right] \Theta_{E}\left(-\frac{1}{2 \pi \sqrt{-1}} \frac{\partial^{2} F_{0}}{\partial \log \mathfrak{q} \partial \vec{a}} \boldsymbol{\beta}\left(d-\frac{r}{2}\right) \mid \tau(\boldsymbol{\beta})\right)
$$

where $\Theta_{E}$ is the Riemann theta function with the characteristic ${ }^{t}\left(\frac{1}{2}, \frac{1}{2}, \ldots, \frac{1}{2}\right)$. (See [14, Appendix B] for convention.) 
As the left hand side is independent of $d$, we have

$$
\begin{aligned}
& \exp \left[-\frac{\partial^{2} F_{0}}{(\partial \log \mathfrak{q})^{2}} \frac{\boldsymbol{\beta}^{2}}{2}\left(d-\frac{r}{2}\right)^{2}\right] \Theta_{E}\left(-\frac{1}{2 \pi \sqrt{-1}} \frac{\partial^{2} F_{0}}{\partial \log \mathfrak{q} \partial \vec{a}} \boldsymbol{\beta}\left(d-\frac{r}{2}\right) \mid \tau(\boldsymbol{\beta})\right) \\
= & \exp \left[-\frac{\partial^{2} F_{0}}{(\partial \log \mathfrak{q})^{2}} \frac{\boldsymbol{\beta}^{2}}{2}\left(d-1-\frac{r}{2}\right)^{2}\right] \Theta_{E}\left(-\frac{1}{2 \pi \sqrt{-1}} \frac{\partial^{2} F_{0}}{\partial \log \mathfrak{q} \partial \vec{a}} \boldsymbol{\beta}\left(d-1-\frac{r}{2}\right) \mid \tau(\boldsymbol{\beta})\right)
\end{aligned}
$$

for $1 \leq d \leq r$. If $d \neq(r+1) / 2$, then this is a differential equation for $F_{0}$ whose solution is determined by the perturbative part, as in Corollary 2.8.

By taking the difference with respect to $d$ again, we recover the contact term equation for the homological partition function (see [13, §7] and [14, §5.3]).

\section{Genus 1 PART}

The coefficients $G, F_{1}$ are called genus 1 part of the partition function in the physics literature. It is known that $F_{1}$ is Gromov-Witten invariants for certain noncompact Calabi-Yau 3 -folds. We determine these in terms of $\tau$ in this section (see (5.6) ).

5.1. Coefficients of $\Lambda^{k}$. We first prepare a result used in the next subsection.

We set $\zeta_{\alpha, \beta} \stackrel{\text { def. }}{=} \frac{1}{1-e^{-\left(a_{\alpha}-a_{\beta}\right) \beta}}$. Then we see that

$$
\begin{aligned}
\frac{1}{1-e^{\left(i \varepsilon_{1}+j \varepsilon_{2}\right) \boldsymbol{\beta}} e^{-\left(a_{\alpha}-a_{\beta}\right) \boldsymbol{\beta}}} & =\frac{1}{1-e^{-\left(a_{\alpha}-a_{\beta}\right) \boldsymbol{\beta}}+e^{-\left(a_{\alpha}-a_{\beta}\right) \boldsymbol{\beta}}\left(1-e^{\left(i \varepsilon_{1}+j \varepsilon_{2}\right) \boldsymbol{\beta}}\right)} \\
& =\frac{1}{1-e^{-\left(a_{\alpha}-a_{\beta}\right) \boldsymbol{\beta}}} \frac{1}{1-\frac{-e^{-\left(a_{\alpha}-a_{\beta}\right) \boldsymbol{\beta}}}{1-e^{-\left(a_{\alpha}-a_{\beta}\right) \boldsymbol{\beta}}}\left(1-e^{\left(i \varepsilon_{1}+j \varepsilon_{2}\right) \boldsymbol{\beta}}\right)} \\
& =\zeta_{\alpha, \beta} \frac{1}{1-\left(1-\zeta_{\alpha, \beta}\right)\left(1-e^{\left(i \varepsilon_{1}+j \varepsilon_{2}\right) \boldsymbol{\beta}}\right)} \\
& =\zeta_{\alpha, \beta} \sum_{k \geq 0}\left(1-\zeta_{\alpha, \beta}\right)^{k}\left(1-e^{\left(i \varepsilon_{1}+j \varepsilon_{2}\right) \boldsymbol{\beta}}\right)^{k}
\end{aligned}
$$

and

$$
\frac{1}{1-e^{\left(i \varepsilon_{1}+j \varepsilon_{2}\right) \boldsymbol{\beta}} e^{-\left(a_{\beta}-a_{\alpha}\right) \boldsymbol{\beta}}}=\left(1-\zeta_{\alpha, \beta}\right) \sum_{k \geq 0} \zeta_{\alpha, \beta}^{k}\left(1-e^{\left(i \varepsilon_{1}+j \varepsilon_{2}\right) \boldsymbol{\beta}}\right)^{k}
$$

Hence

$$
Z^{\text {inst }} \in\left(\mathbb{C}\left[\zeta_{\alpha, \beta}\right]\left[\left[\varepsilon_{1}, \varepsilon_{2}\right]\right] \otimes \mathbb{C}\left(\varepsilon_{1}, \varepsilon_{2}\right)\right)\left[\left[\prod_{\alpha<\beta} \zeta_{\alpha, \beta} \Lambda\right]\right]
$$

In particular,

$$
F^{\text {inst }} \in \mathbb{C}\left[\zeta_{\alpha, \beta}\right]\left[\left[\prod_{\alpha<\beta} \zeta_{\alpha, \beta} \Lambda\right]\right]\left[\left[\varepsilon_{1}, \varepsilon_{2}\right]\right]
$$

Since

$$
\begin{aligned}
\frac{\partial \zeta_{\alpha, \beta}^{n}}{\partial a_{l}} & =n \zeta_{\alpha, \beta}^{n-1} \frac{\partial \zeta_{\alpha, \beta}}{\partial a_{l}} \\
& =n \zeta_{\alpha, \beta}^{n-1} \frac{-e^{-\left(a_{\alpha}-a_{\beta}\right) \boldsymbol{\beta}}}{\left(1-e^{-\left(a_{\alpha}-a_{\beta}\right) \boldsymbol{\beta}}\right)^{2}} \frac{\partial\left(a_{\alpha}-a_{\beta}\right) \boldsymbol{\beta}}{\partial a_{l}} \\
& =\boldsymbol{\beta} n \zeta_{\alpha, \beta}^{n}\left(1-\zeta_{\alpha, \beta}\right)\left(e_{\alpha}-e_{\beta}, e_{l}\right),
\end{aligned}
$$


we also have

$$
\frac{\partial}{\partial a_{l}} \frac{\partial F_{0}^{\text {inst }}}{\partial a_{m}} \in \mathbb{C}\left[\zeta_{\alpha, \beta}\right]\left[\left[\prod_{\alpha<\beta} \zeta_{\alpha, \beta} \Lambda\right]\right]
$$

5.2. Genus 1 parts as modular forms. Assume that $0<k<r$. By Theorem 2.4(2), we get

$$
\sum_{\{\vec{k}\}=-k / r} Z\left(\varepsilon_{1}, \varepsilon_{2}-\varepsilon_{1}, \vec{a}+\varepsilon_{1} \vec{k} ; t_{1}^{d-\frac{r}{2}} \mathfrak{q}, \boldsymbol{\beta}\right) Z\left(\varepsilon_{1}-\varepsilon_{2}, \varepsilon_{2}, \vec{a}+\varepsilon_{2} \vec{k} ; t_{2}^{d-\frac{r}{2}} \mathfrak{q}, \boldsymbol{\beta}\right)=0
$$

for $0<d<r$.

As in the derivation of (4.15) we have

$$
\begin{aligned}
0=\sum_{\{\vec{k}\}=-\frac{k}{r}} \exp [ & -\frac{\partial^{2} F_{0}}{\partial a^{l} \partial a^{m}} \frac{k^{l} k^{m}}{2}-\frac{\partial^{2} F_{0}}{\partial \log \mathfrak{q} \partial a^{l}} \boldsymbol{\beta}\left(d-\frac{r}{2}\right) k^{l}-\frac{\partial^{2} F_{0}}{(\partial \log \mathfrak{q})^{2}} \frac{\boldsymbol{\beta}^{2}}{2}\left(d-\frac{r}{2}\right)^{2}+\frac{\partial H}{\partial a^{l}} k^{l} \\
& +\left(\varepsilon_{1}+\varepsilon_{2}\right)\left\{-\frac{\partial^{3} F_{0}}{\partial a^{l} \partial a^{m} \partial a^{n}} \frac{k^{l} k^{m} k^{n}}{3 !}-\frac{\partial^{3} F_{0}}{\partial a^{l} \partial a^{m} \partial \log \mathfrak{q}} \frac{k^{l} k^{m}}{2 !} \boldsymbol{\beta}\left(d-\frac{r}{2}\right)\right. \\
& -\frac{\partial^{3} F_{0}}{\partial a^{l} \partial(\log \mathfrak{q})^{2}} \frac{k^{l}}{2 !} \boldsymbol{\beta}^{2}\left(d-\frac{r}{2}\right)^{2}-\frac{\partial^{3} F_{0}}{\partial(\log \mathfrak{q})^{3}} \frac{1}{3 !} \boldsymbol{\beta}^{3}\left(d-\frac{r}{2}\right)^{3} \\
& \left.\left.+\frac{\partial\left(G+F_{1}\right)}{\partial a^{l}} k^{l}+\frac{\partial\left(G+F_{1}\right)}{\partial \log \mathfrak{q}} \boldsymbol{\beta}\left(d-\frac{r}{2}\right)\right\}+\cdots\right] .
\end{aligned}
$$

Hence we have

$$
\begin{aligned}
0=\sum_{\{\vec{k}\}=-\frac{k}{r}}(-1)^{-\langle\vec{k}, \vec{\rho}\rangle} \exp [ & -\frac{\partial^{2} F_{0}}{\partial a^{l} \partial a^{m}} \frac{k^{l} k^{m}}{2}-\frac{\partial^{2} F_{0}}{\partial \log \mathfrak{q} \partial a^{l}} \boldsymbol{\beta}\left(d-\frac{r}{2}\right) k^{l} \\
& +\left(\varepsilon_{1}+\varepsilon_{2}\right)\left\{-\frac{\partial^{3} F_{0}}{\partial a^{l} \partial a^{m} \partial a^{n}} \frac{k^{l} k^{m} k^{n}}{3 !}-\frac{\partial^{3} F_{0}}{\partial a^{l} \partial a^{m} \partial \log \mathfrak{q}} \frac{k^{l} k^{m}}{2 !} \boldsymbol{\beta}\left(d-\frac{r}{2}\right)\right. \\
& \left.\left.-\frac{\partial^{3} F_{0}}{\partial a^{l} \partial(\log \mathfrak{q})^{2}} \frac{k^{l}}{2 !} \boldsymbol{\beta}^{2}\left(d-\frac{r}{2}\right)^{2}+\frac{\partial\left(G+F_{1}\right)}{\partial a^{l}} k^{l}\right\}+\cdots\right] .
\end{aligned}
$$

Setting $\varepsilon_{1}=\varepsilon_{2}=0$, we have

$$
0=\sum_{\{\vec{k}\}=-\frac{k}{r}}(-1)^{-\langle\vec{k}, \vec{\rho}\rangle} \exp \left[-\frac{\partial^{2} F_{0}}{\partial a^{l} \partial a^{m}} \frac{k^{l} k^{m}}{2}-\frac{\partial^{2} F_{0}}{\partial \log \mathfrak{q} \partial a^{l}} \boldsymbol{\beta}\left(d-\frac{r}{2}\right) k^{l}\right] .
$$

By looking at the coefficient of $\varepsilon_{1}+\varepsilon_{2}$, we get

$$
\begin{aligned}
0=\sum_{\{\vec{k}\}=-\frac{k}{r}}(-1)^{-\langle\vec{k}, \vec{\rho}\rangle} \exp & {\left[-\frac{\partial^{2} F_{0}}{\partial a^{l} \partial a^{m}} \frac{k^{l} k^{m}}{2}-\frac{\partial^{2} F_{0}}{\partial \log \mathfrak{q} \partial a^{l}} \boldsymbol{\beta}\left(d-\frac{r}{2}\right) k^{l}\right] } \\
& \times\left\{-\frac{\partial^{3} F_{0}}{\partial a^{l} \partial a^{m} \partial a^{n}} \frac{k^{l} k^{m} k^{n}}{3 !}-\frac{\partial^{3} F_{0}}{\partial a^{l} \partial a^{m} \partial \log \mathfrak{q}} \frac{k^{l} k^{m}}{2 !} \boldsymbol{\beta}\left(d-\frac{r}{2}\right)\right. \\
& \left.-\frac{\partial^{3} F_{0}}{\partial a^{l} \partial(\log \mathfrak{q})^{2}} \frac{k^{l}}{2 !} \boldsymbol{\beta}^{2}\left(d-\frac{r}{2}\right)^{2}+\frac{\partial\left(G+F_{1}\right)}{\partial a^{l}} k^{l}\right\} .
\end{aligned}
$$

From now on, we assume that $r=2$ and $d=1$. We set $\vec{a}:=(-a, a)$. Then 


$$
\sum_{\{\vec{k}\}=-\frac{1}{2}}(-1)^{-\langle\vec{k}, \rho\rangle} e^{\pi \sqrt{-1} \tau k^{2}}\left(-\frac{\partial^{3} F_{0}}{\partial a^{3}} \frac{k^{3}}{3 !}+\frac{\partial\left(F_{1}+G\right)}{\partial a} k\right)=0 .
$$

This equation can be rewritten as

$$
\frac{\partial}{\partial a}\left(G+F_{1}\right)=-\frac{1}{3} \frac{\partial}{\partial a} \log \left(\frac{\partial}{\partial \xi} \theta_{11}(\xi \mid \tau)_{\mid \xi=0}\right) .
$$

By Jacobi's derivative formula, we get

$$
G+F_{1}=-\frac{1}{3} \log \left(-2 \pi q^{\frac{1}{4}} \prod_{d=1}^{\infty}\left(1-q^{2 d}\right)^{3}\right)+C
$$

where $C$ is a function on $\Lambda$. Combining this with (4.15) (with $r=2, d=1$ ), we see that

$$
\exp \left(F_{1}\right)=\frac{C^{\prime}}{\eta(\tau / 2)}
$$

where $C^{\prime}$ is a function on $\Lambda$. On the other hand,

$$
\exp \left(F_{1}\right) \eta(\tau / 2) \in \mathbb{C}\left[\zeta_{1,2}\right]\left[\left[\zeta_{1,2} \Lambda\right]\right]
$$

Proof. We note the following relations:

$$
\begin{gathered}
F_{1}=\frac{1}{12} \log \left(\frac{1-e^{-a \boldsymbol{\beta}}}{\boldsymbol{\beta} \Lambda}\right)+\frac{1}{12} \log \left(\frac{1-e^{a \boldsymbol{\beta}}}{\boldsymbol{\beta} \Lambda}\right)+F_{1}^{\text {inst }}, \\
\log \eta(\tau / 2)^{-1}=-\frac{1}{24} \pi \sqrt{-1} \tau+O(q), \\
\tau=\frac{\sqrt{-1}}{\pi} 2\left(\log \left(\frac{1-e^{-a \boldsymbol{\beta}}}{\boldsymbol{\beta} \Lambda}\right)+\log \left(\frac{1-e^{a \boldsymbol{\beta}}}{\boldsymbol{\beta} \Lambda}\right)\right)-\frac{1}{2 \pi \sqrt{-1}} \frac{\partial^{2} F_{0}^{\text {inst }}}{\partial a^{2}} .
\end{gathered}
$$

Since $F_{1}^{\text {inst }}, \partial^{2} F_{0}^{\text {inst }} / \partial a^{2} \in \mathbb{C}\left[\zeta_{1,2}\right]\left[\left[\zeta_{1,2} \Lambda\right]\right](\underline{(5.3)}$, (15.5) $)$, we get our claim.

Hence $C^{\prime}$ is a constant. By the same proof, we also see that $C^{\prime}=1$. Therefore we get the following equalities:

$$
\begin{aligned}
& \exp \left(F_{1}\right)=\frac{1}{\eta(\tau / 2)} \\
& \exp (G)=q^{-1 / 24} \prod_{d=1}^{\infty}\left(1-q^{2 d-1}\right) .
\end{aligned}
$$

\section{Appendix A. NORMAL VARIETIES}

Lemma A.1. Let $X$ be a normal variety over $\mathbb{C}$. Let $f: X \rightarrow Y$ be a proper and surjective morphism to a normal variety $Y$ and $g: X \rightarrow Z$ a morphism to a variety $Z$. Assume that $g\left(f^{-1}(y)\right)$ is a point for every $y \in Y$. Then we have a unique morphism $h: Y \rightarrow Z$ such that $h \circ f=g$.

Proof. We note that $f$ is factorized $X \hookrightarrow X \times Z \stackrel{f \times 1_{Z}}{\rightarrow} Y \times Z \rightarrow Y$. Since $f \times 1_{Z}$ is proper, $W:=\left(f \times 1_{Z}\right)(X)$ is a closed subvariety of $Y \times Z$. Since $X \rightarrow W$ is surjective and $X \rightarrow W \rightarrow Y$ is proper, $W \rightarrow Y$ is also proper. Since $g\left(f^{-1}(y)\right)$ is a point for every $y \in Y, W \rightarrow Y$ is injective. Hence it is finite and birational. Since $Y$ is normal, we conclude that $W \cong Y$. Therefore we have a desired morphism $h: Y \rightarrow Z$. 
Corollary A.2. Let $X$ be a normal variety and $R$ an equivalence relation on $X(\mathbb{C})$ (the set of $\mathbb{C}$-valued points of $X)$. Assume that there is a proper map $g: X \rightarrow Y$ such that $Y(\mathbb{C})=X(\mathbb{C}) / R$. Then there is a proper morphism $f: X \rightarrow M$ such that $M$ is normal, $M(\mathbb{C})=X(\mathbb{C}) / R$ and there is a unique morphism $h: M \rightarrow Y$ with $g=h \circ f$. We call $M$ the quotient of $X$ by $R$.

\section{Appendix B. Polylogarithms}

B.1. We define the $k$ th polylogarithm inductively by

$$
\begin{gathered}
\operatorname{Li}_{k}(0)=0, \quad \operatorname{Li}_{0}(w)=\frac{w}{1-w}, \\
\frac{d}{d w} \operatorname{Li}_{k}(w)=\frac{1}{w} \operatorname{Li}_{k-1}(w),
\end{gathered}
$$

where $w<1$. We have

$$
\operatorname{Li}_{1}(w)=\int \frac{1}{w} \operatorname{Li}_{0}(w) d w=-\log (1-w)
$$

We also have

$$
\begin{gathered}
\operatorname{Li}_{-1}(w)=w \frac{d}{d w} \operatorname{Li}_{0}(w)=\frac{w}{(1-w)^{2}}, \quad \operatorname{Li}_{-2}(w)=w \frac{d}{d w} \operatorname{Li}_{-1}(w)=\frac{w+w^{2}}{(1-w)^{3}}, \\
\operatorname{Li}_{-k}(w)=\frac{w P_{k}(w)}{(1-w)^{k+1}} \quad\left(k \in \mathbb{Z}_{\geq 0}\right),
\end{gathered}
$$

where $P_{0}(w)=1$ and $P_{k}(w)$ is a polynomial of degree $k-1$ for $k>0$, satisfying the recursive system

$$
P_{k+1}(w)=(1+k w) P_{k}(w)+w(1-w) P_{k}^{\prime}(w) .
$$

In particular, $\operatorname{Li}_{-k}(w)$ is defined for $w \neq 1$. For $|w|<1, \operatorname{Li}_{k}$ can be expressed by a series

$$
\operatorname{Li}_{k}(w)=\sum_{n=1}^{\infty} \frac{w^{n}}{n^{k}}
$$

B.2. Inversion formulas. We need to relate $\operatorname{Li}_{k}(1 / w)$ to $\operatorname{Li}_{k}(w)$. For $k=-l$ with $l>0$, we have

$$
\begin{gathered}
\operatorname{Li}_{0}(1 / w)=\frac{1 / w}{(1-1 / w)}=\frac{1}{w-1}=-1-\operatorname{Li}_{0}(w), \\
\operatorname{Li}_{-l}\left(\frac{1}{w}\right)=\left(\frac{1}{w} \frac{d}{d(1 / w)}\right)^{l} \operatorname{Li}_{0}\left(\frac{1}{w}\right)=-\left(-w \frac{d}{d w}\right)^{l} \operatorname{Li}_{01}(w)=(-1)^{l-1} \operatorname{Li}_{-l}(w) .
\end{gathered}
$$

Next consider $\operatorname{Li}_{1}(w)=-\log (1-w)$. We need to specify how we take the branch of $\log$. We set $w=e^{-y}$ with $y>0$. Then $\operatorname{Li}_{1}\left(e^{-y}\right)$ is defined. We define $\operatorname{Li}_{1}\left(e^{-y}\right)$ with $y<0$ by analytic continuation. Then

$$
\operatorname{Li}_{1}\left(e^{y}\right)=\operatorname{Li}_{1}\left(e^{-y}\right)-y-\pi \sqrt{-1} .
$$

If $2 \pi>y>0$, then by integrating

$$
\log \left(1-e^{-y}\right)=\log y-\frac{1}{2} y+\frac{B_{1}}{2 \cdot 2 !} y^{2}-\frac{B_{2}}{4 \cdot 4 !} y^{4}-\cdots,
$$

we have

$$
\mathrm{Li}_{2}\left(e^{-y}\right)=\frac{\pi^{2}}{6}+(y \log y-y)-\frac{y^{2}}{4}+\frac{B_{1}}{2 \cdot 3 \cdot 2 !} y^{3}-\frac{B_{2}}{4 \cdot 5 \cdot 4 !} y^{5}-\cdots
$$


Note that

$$
-\frac{y^{2}}{4}+\frac{B_{1}}{2 \cdot 3 \cdot 2 !} y^{3}-\frac{B_{2}}{4 \cdot 5 \cdot 4 !} y^{5}-\cdots .
$$

converges for $|y|<2 \pi$. We define $\operatorname{Li}_{2}\left(e^{-y}\right), y<0$ by analytic continuation. So

$$
\mathrm{Li}_{2}\left(e^{-y}\right)=\frac{\pi^{2}}{6}+(y \log y-y)-\frac{y^{2}}{4}+\frac{B_{1}}{2 \cdot 3 \cdot 2 !} y^{3}-\frac{B_{2}}{4 \cdot 5 \cdot 4 !} y^{5}-\cdots .
$$

for $|y|<2 \pi$. Hence

$$
\begin{aligned}
\mathrm{Li}_{2}\left(e^{y}\right)+\mathrm{Li}_{2}\left(e^{-y}\right) & =\frac{\pi^{2}}{3}-y \log (-y)+y \log y-\frac{y^{2}}{2} \\
& =\frac{\pi^{2}}{3}-y \pi \sqrt{-1}-\frac{y^{2}}{2}
\end{aligned}
$$

for $y>0$. By integrating (B.5), we have

$$
\operatorname{Li}_{3}\left(e^{-y}\right)=\zeta(3)-\frac{\pi^{2}}{6} y-\frac{y^{2}}{2} \log y+\frac{3}{4} y^{2}+\frac{y^{3}}{12}-\left(\frac{B_{1}}{2 \cdot 3 \cdot 4 \cdot 2 !} y^{4}-\frac{B_{2}}{4 \cdot 5 \cdot 6 \cdot 4 !} y^{6}-\cdots\right)
$$

for $|y|<2 \pi$. Hence we have

$$
\begin{aligned}
\operatorname{Li}_{3}\left(e^{y}\right) & =\operatorname{Li}_{3}\left(e^{-y}\right)+\frac{\pi^{2}}{3} y+\frac{1}{2} y^{2} \log y-\frac{1}{2} y^{2} \log (-y)-\frac{y^{3}}{6} \\
& =\operatorname{Li}_{3}\left(e^{-y}\right)+\frac{\pi^{2}}{3} y-\frac{1}{2} y^{2} \pi \sqrt{-1}-\frac{y^{3}}{6}
\end{aligned}
$$

for $y>0$.

B.3. Limit. We have

$$
\lim _{\beta \rightarrow 0} \beta^{k+1} \mathrm{Li}_{-k}\left(e^{-\boldsymbol{\beta} x}\right)=x^{-k-1} P_{k}(1)=k ! x^{-k-1}
$$

for $k \in \mathbb{Z}_{\geq 0}$.

We have

$$
\operatorname{Li}_{1}\left(e^{-\boldsymbol{\beta} x}\right)+\log (\boldsymbol{\beta} \Lambda)=-\log \left(\frac{1-e^{-\boldsymbol{\beta} x}}{\boldsymbol{\beta} \Lambda}\right) \underset{\boldsymbol{\beta} \rightarrow 0}{\longrightarrow}-\log \left(\frac{x}{\Lambda}\right)
$$

Then

$$
\begin{aligned}
& \int_{0}^{x} \operatorname{Li}_{1}\left(e^{-\boldsymbol{\beta} x^{\prime}}\right)+\log (\boldsymbol{\beta} \Lambda) d x^{\prime}=-\frac{1}{\boldsymbol{\beta}}\left(\operatorname{Li}_{2}\left(e^{-\boldsymbol{\beta} x}\right)-\frac{\pi^{2}}{6}\right)+x \log (\boldsymbol{\beta} \Lambda) \\
& \underset{\boldsymbol{\beta} \rightarrow 0}{\longrightarrow}-\int_{0}^{x} \log \left(\frac{x^{\prime}}{\Lambda}\right) d x^{\prime}=-x \log \left(\frac{x}{\Lambda}\right)+x .
\end{aligned}
$$

Furthermore

$$
\begin{aligned}
\int_{0}^{x} & -\frac{1}{\boldsymbol{\beta}}\left(\operatorname{Li}_{2}\left(e^{-\boldsymbol{\beta} x^{\prime}}\right)-\frac{\pi^{2}}{6}\right)+x^{\prime} \log (\boldsymbol{\beta} \Lambda) d x^{\prime} \\
& =\frac{1}{\boldsymbol{\beta}^{2}}\left(\operatorname{Li}_{3}\left(e^{-\boldsymbol{\beta} x}\right)-\zeta(3)\right)+\frac{x^{2}}{2} \log (\boldsymbol{\beta} \Lambda)+\frac{\pi^{2} x}{6 \boldsymbol{\beta}} \\
\underset{\boldsymbol{\beta} \rightarrow 0}{\longrightarrow} & \int_{0}^{x}\left[-x^{\prime} \log \left(\frac{x^{\prime}}{\Lambda}\right)+x^{\prime}\right] d x^{\prime}=-\frac{1}{2} x^{2} \log \left(\frac{x}{\Lambda}\right)+\frac{3}{4} x^{2} .
\end{aligned}
$$




\section{REFERENCES}

[1] M.F. Atiyah and G.B. Segal, The index of elliptic operators, II, Ann. of Math., 87 (1968), 531-545.

[2] J. Bryan, Symplectic geometry and the relative Donaldson invariants of $\overline{\mathbb{C P}}^{2}$, Forum Math. 9 (1997), $325-365$.

[3] W. Crawley-Boevey, Normality of Marsden-Weinstein reductions for representations of quivers, Math. Ann. 325 (2003), 55-79.

[4] D. Edidin and W. Graham, Equivariant intersection theory, Invent. Math. 131 (1998), 595-634.

[5] L. Göttsche, H. Nakajima and K. Yoshioka, in preparation.

[6] A. Gorsky, A. Marshakov, A. Mironov and A. Morozov, RG equations from Whitham hierarchy, Nucl. Phys. B 527 (1998), 690-716; hep-th/9802007

[7] Y. Kawamata, K. Matsuda and K. Matsuki, Introduction to the minimal model problem, Algebraic geometry, Sendai, 1985, 283-360, Adv. Stud. Pure Math., 10, North-Holland, Amsterdam, 1987

[8] J. Li, Algebraic geometric interpretation of Donaldson's polynomial invariants, J. Differential Geom. 37 (1993) 417-466.

[9] — Compactification of moduli of vector bundles over algebraic surfaces, Collection of papers on geometry, analysis and mathematical physics, World Sci. Publishing, River Edge, NJ, (1997) 98-113.

[10] M. Mariño, The uses of Whitham hierarchies, Progr. Theoret. Phys. Suppl. No. 135 (1999), 29-52; hep-th/9905053

[11] M. Maruyama, Instantons and parabolic sheaves, Geometry and analysis (Bombay, 1992), 245-267, Tata Inst. Fund. Res., Bombay, 1995.

[12] M. Maruyama and K. Yokogawa, Moduli of parabolic stable sheaves, Math. Ann. 293 (1992), 77-99

[13] H. Nakajima and K. Yoshioka, Instanton counting on blowup. I. 4-dimensional pure gauge theory, math.AG/0306198 Invent. Math. to appear

[14] Lectures on instanton counting, in Algebraic Structures and Moduli Spaces, CRM Proceedings \& Lecture Notes 38, AMS, 2004, 31-101; math.AG/0311058

[15] N. Nekrasov, Seiberg-Witten prepotential from instanton counting, Adv. Theor. Math. Phys. 7 (2003), no. $5,831-864 ;$ hep-th/0206161

[16] N. Nekrasov and A. Okounkov, Seiberg-Witten prepotential and random partitions, preprint, hep-th/0306238

[17] N. Seiberg and E. Witten, Electric-magnetic duality, monopole condensation, and confinement in $N=2$ supersymmetric Yang-Mills theory Nuclear Phys. B 426 (1994), 19-52; Erratum, Nuclear Phys. B 430 (1994), 485-486.

[18] R. Thomason, Une formule de Lefschetz en K-théorie équivariante algébrique, Duke Math. 68 (1992), $447-462$.

[19] K. Yoshioka: A note on a paper of J.-M. Drézet on the local factoriality of some moduli spaces, Internat. J. Math. 7 (1996), 843-858

Department of Mathematics, Kyoto University, Kyoto 606-8502, Japan

E-mail address: nakajima@math.kyoto-u.ac.jp

Department of Mathematics, Faculty of Science, Kobe University, Kobe 657-8501, Japan

E-mail address: yoshioka@math.kobe-u.ac.jp 